Portland State University

PDXScholar

Physics Faculty Publications and Presentations

Physics

2020

Polarization in the Production of the Antihydrogen Ion

Casey A. Yazejian

Portland State University

Jack C. Straton

Portland State University, straton@pdx.edu

Follow this and additional works at: https://pdxscholar.library.pdx.edu/phy_fac

Part of the Atomic, Molecular and Optical Physics Commons

Let us know how access to this document benefits you.

Citation Details

Yazejian, C. A., \& Straton, J. C. (2020). Polarization in the production of the antihydrogen ion. The European Physical Journal D, 74(7), 1-16.

This Article is brought to you for free and open access. It has been accepted for inclusion in Physics Faculty Publications and Presentations by an authorized administrator of PDXScholar. Please contact us if we can make this document more accessible: pdxscholar@pdx.edu. 


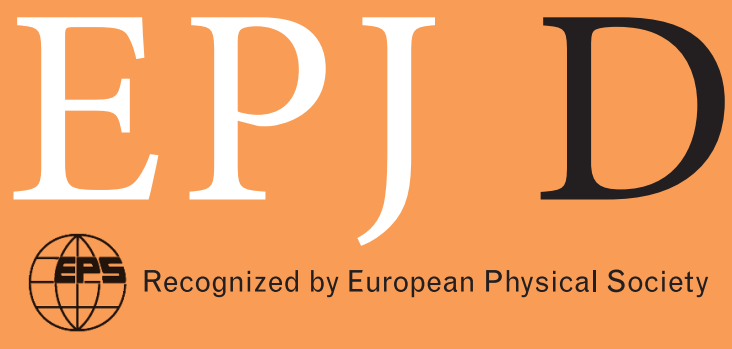

\section{Atomic, Molecular, Optical and Plasma Physics}

Eur. Phys. J. D (2020) 74: 156

DOI: $10.1140 /$ epjd/e2020-100548-7

\section{Polarization in the production of the antihydrogen ion}

Casey A. Yazejian, and Jack C. Straton
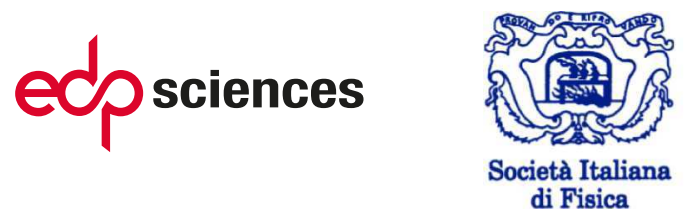

算 Springer 


\title{
Polarization in the production of the antihydrogen ion ${ }^{\star}$
}

\author{
Casey A. Yazejian and Jack C. Straton ${ }^{\mathrm{a}}$ \\ Department of Physics, Portland State University, Portland, OR 97207-0751, USA
}

Received 31 October 2019 / Received in final form 1 May 2020

Published online 23 July 2020

(c) EDP Sciences / Società Italiana di Fisica / Springer-Verlag GmbH Germany, part of Springer Nature, 2020

\begin{abstract}
We provide estimates of both the cross section and rate coefficient for the radiative attachment of a second positron to create the $\overline{\mathrm{H}}^{+}$ion, $\overline{\mathrm{H}}(1 s)+e^{+} \rightarrow \overline{\mathrm{H}}^{+}\left(1 s^{2}{ }^{1} S^{e}\right)+\hbar \omega$, for which the polarization of the initial state $\overline{\mathrm{H}}(1 s)$ is taken into account. We show how to analytically integrate the resulting six-dimensional, three-body integrals for wave functions composed of explicitly correlated exponentials, a result that may be extended to Hylleraas wave functions. We extend Bhatia's polarization results for the equivalent matter problem down to the low temperatures required for the Gravitational Behaviour of Antihydrogen at Rest (GBAR) experiment at CERN. The two-electron polarization cross-term is of intrinsic interest because it has every appearance of being singular at the origin, but non-singular when integrated numerically. We show that conventional approaches lead to a final integral with two singular terms that may be made to cancel in lowest order. However, higher-order terms in such approaches defy analytical integration. We use an integro-differential transform based on Gaussian transforms to bypass this blockage to yield a fully analytic result. Even in this method, one avoids the singular form only by integrating out the radial integrals before solving the second Gaussian integral.
\end{abstract}

\section{Introduction}

For more than a decade, production of antihydrogen $(\overline{\mathrm{H}})$ atoms at the Antiproton Decelerator (AD) facility at CERN [1] has provided the foundation for a variety of experiments (e.g., [2-4]). The ALPHA and ATRAP collaborations now trap small numbers of these anti-atoms in specialized magnetic minimum neutral atom traps [5-7], and confinement times of many minutes are now possible at ALPHA [8]. This has led to spectroscopic [9] measurements for $\overline{\mathrm{H}}$, an experimental limit on its charge [10], and even a rough limit on the gravitational interaction of the anti-atom [11].

The GBAR collaboration [12-14] has built on the latter idea to measure the gravitational attraction of matter versus antimatter using neutral $\overline{\mathrm{H}}$ atoms, but these are difficult to cool sufficiently. They plan to form the antihydrogen ion $\overline{\mathrm{H}}^{+}$as an intermediate step because its net charge allows for sympathetic cooling with a mixture of positively charged ions of ordinary matter like $\mathrm{Be}^{+}$. After cooling, the extra positron would then be stripped off for studies of the gravitational interaction of the anti-atom [12-14]. Prior papers $[15,16]$ have calculated the cross section and

* Contribution to the Topical Issue "Low-Energy Positron and Positronium Physics and Electron-Molecule Collisions and Swarms (POSMOL 2019)", edited by Michael Brunger, David Cassidy, Saša Dujko, Dragana Maric, Joan Marler, James Sullivan, Juraj Fedor.

a e-mail: straton@pdx.edu rate coefficient for the radiative attachment of a second positron to create the $\overline{\mathrm{H}}^{+}$ion,

$$
\overline{\mathrm{H}}(1 s)+e^{+} \rightarrow \overline{\mathrm{H}}^{+}\left(1 s^{2}{ }^{1} S^{e}\right)+\hbar \omega,
$$

utilizing both [15] the effective range wave function of Ohmura and Ohmura [17] as well as [16] a fully twopositron 200-term wave function [18] composed of explicitly correlated exponentials of the kind introduced by Thakkar and Smith [19]. These extend to temperatures lower than Bhatia's [20] results. Bhatia's results, however, include both long-range polarization effects and shortrange correlations arising from an optical potential. It is important to know how those effects manifest at these lower temperatures and the present paper includes the former but only a modest amount of the latter.

\section{The cross section}

The radiative attachment cross section can be most easily found from the photoionization cross section via the principle of detailed balance (see, e.g., [21]), as was done by Drake [22] and then Jacobs et al. [23], who applied the principle of detailed balance to obtain the radiative attachment coefficient (for an electron) to form the $\left(2 p^{2}{ }^{3} P^{e}\right)$ metastable $\mathrm{H}^{-}$state from $\mathrm{H}(2 s, 2 p)$. Although Chandrasekhar $[24,25]$ was not the first to calculate the cross section for photoionization (also called photodetachment), $\sigma_{\mathrm{PI}}$, of the equivalent matter problem, many 
researchers follow his lead. For instance, Ghoshal and and Ho [26] re-expressed Chandrasekhar's form of the (length gauge) cross section in atomic units,

$$
\begin{aligned}
\sigma_{\mathrm{PI}} & =\frac{2 p \omega \alpha a_{0}^{2}}{3}\left|\left\langle\psi_{f}\left|\hat{k} \cdot\left(\mathbf{r}_{1}+\mathbf{r}_{2}\right)\right| \psi_{i}\right\rangle\right|^{2} \\
& =6.81156 \times 10^{-20} \mathrm{~cm}^{2} k\left(k^{2}+2 I\right)\left|\left\langle\psi_{f}\left|z_{1}+z_{2}\right| \psi_{i}\right\rangle\right|^{2} \\
& \equiv 6.81156 \times 10^{-20} \mathrm{~cm}^{2} k\left(k^{2}+2 I\right)\left|\mu_{\mathrm{PI}}\right|^{2}
\end{aligned}
$$

where $\alpha \approx 1 / 137$ is the fine structure constant, $a_{0}$ the Bohr radius. Conservation laws give the energy $\hbar \omega$ of the ionized photon in terms of the sum of the magnitude of the momentum of the detached electron $p=\hbar k$ and the energy difference between initial and final bound states (called the electron affinity $I \equiv \gamma_{0}^{2} / 2$ ), by

$$
2 \hbar \omega=2(2 \pi h \nu)=2 \frac{h c}{\lambda}=2\left(\frac{\hbar^{2} k^{2}}{2}+I\right) \equiv\left(p^{2}+\gamma_{0}^{2}\right) .
$$

In equation (2), the matrix element for photodetachment is

$$
\mu_{\mathrm{PI}}=\int d \tau \psi_{f}^{*}\left(z_{1}+z_{2}\right) \psi_{i},
$$

which includes initial- and final-state wave functions that represent two-positron (or two-electron) states. Whereas one-positron states can be represented by an analytic function, two-positron states can be represented only approximately.

Our goal in doing these calculations has been to provide the GBAR collaboration with increasingly accurate estimates of the rate coefficient so that they can decide if this approach will bear fruit for their experiment. Thus, in our first paper [15] we represented the final-state wave function as the product of a plane wave multiplied by an antihydrogen ground state wave function,

$$
\psi_{f}=\frac{1}{\sqrt{2 \pi}}\left(e^{i k z_{1}-r_{2}}+e^{i k z_{2}-r_{1}}\right) .
$$

The initial-state $\overline{\mathrm{H}}^{+}$ion is so weakly bound that there is but one bound state. As a first approximation, one may imagine that the state consists of one positron bound to the antiproton in the antihydrogen ground state, while the second positron is at such a large distance that the bound positron fully screens the antiproton charge so that the second positron feels no force. Hence the radial wave function of the weakly bound positron $\psi_{\mathrm{eff}}(r)$ satisfies the Schrödinger equation

$$
r \frac{d^{2}}{d r^{2}} \psi_{\mathrm{eff}}(r)+2 \frac{d}{d r} \psi_{\mathrm{eff}}(r)=r \psi_{\mathrm{eff}}(r) \gamma_{0}^{2},
$$

whose solution is

$$
\psi_{\mathrm{eff}}(r)=\frac{C}{r} \exp \left(-\gamma_{0} r\right) .
$$

Thus, in this first approximation we have a nearly unbound positron becoming a positron truly in the continuum due to the absorption of a photon. In this effective range theory of Ohmura and Ohmura [17], the antihydrogen (hydrogen) atom is a spectator and we have now a one-positron matrix element for (4).

The next step in our set of calculations for GBAR was to utilize a very accurate two-positron initial-state wave function of the sort Thakkar and Smith [19] introduced made up of explicitly correlated exponentials,

$$
\psi_{i}\left(r_{1}, r_{2}, r_{12}\right)=\frac{1}{\sqrt{2}}\left(1-\hat{P}_{12}\right) \sum_{k} c_{k} e^{-\alpha_{k} r_{1}-\beta_{k} r_{2}-\gamma_{k} r_{12}},
$$

where $\hat{P}_{12}$ is the permutation operator for the two identical positrons. The parameters in the exponentials are generated in a quasi-random fashion,

$$
\begin{aligned}
& \alpha_{k}=\eta\left(\left(A_{2}-A_{1}\right) \frac{1}{2}\langle k(k+1)\rangle \sqrt{2}+A_{1}\right) \\
& \beta_{k}=\eta\left(\left(B_{2}-B_{1}\right) \frac{1}{2}\langle k(k+1)\rangle \sqrt{3}+B_{1}\right) \\
& \gamma_{k}=\eta\left(\left(G_{2}-G_{1}\right) \frac{1}{2}\langle k(k+1)\rangle \sqrt{5}+G_{1}\right),
\end{aligned}
$$

where $\langle x\rangle$ denotes the fractional part of $x$. For the twopositron 200-term wave function we used, these parameters are [18]: $A_{1}=0.22277, A_{2}=1.58047, B_{1}=$ $0.98603, B_{2}=1.33237, G_{1}=-0.16261, G_{2}=0.76359$, and $\eta=1+1.42 \times 10^{-7}$. Because the optimization algorithm is not perfect, one must scale the wave function with $\eta$ very slightly different from one so that it satisfies the virial theorem. The coefficients $c_{k}$ are found by diagonalizing the Hamiltonian matrix in order to minimize the ground state energy and are then normalized. The quality of this wave function may be seen in the energy it produces: 0.5277510163 a.u. agreeing with the 0.527751016 a.u. benchmark result of Pekeris [27,28].

Our results from using this highly accurate wave function for the initial state, were about $4 \%$ lower than those from using the very approximate wave function from the effective range theory of Ohmura and Ohmura [17]. That is, an initial-state wave function for a very-slightly-bound system that is fully correlated at all inter-positron distances gave results that were only a modest improvement over an initial-state wave function with zero correlation at any inter-positron distance apart from the assumption that it was most often at a large distance.

These results using a highly accurate wave function for the initial state, however, differed significantly from Bhatia's [20] photoionization results at high wave numbers and from his rate coefficient at high temperatures. Clearly there was a need to improve the final-state wave function (5). We chose to focus in this work on the polarization effects that a continuum positron has on the antihydrogen bound state.

\section{Polarization}

In 1957 Temkin [29] introduced a means to account for the polarization of an atom by the presence of a free electron, 
which for the present problem modifies the antihydrogen ground state as

$$
\begin{aligned}
& \psi_{f}\left(r_{1}, r_{2}, \cos \theta_{12}\right) \\
& =\frac{1}{\sqrt{2}}\left[\left(\frac{\lambda^{3 / 2}}{\sqrt{\pi}} e^{-\lambda r_{1}}-\frac{1}{\sqrt{\pi} \lambda^{1 / 2}}\right.\right. \\
& \left.\quad \times \frac{\epsilon\left(r_{1}, r_{2}\right)\left(\frac{\lambda r_{1}^{3}}{2}+r_{1}^{2}\right) \cos \theta_{12} e^{-\lambda r_{1}}}{r_{1} r_{2}^{2}}\right) e^{i k z_{2}} \\
& \left.+\left(\frac{\lambda^{3 / 2}}{\sqrt{\pi}} e^{-\lambda r_{2}}-\frac{1}{\sqrt{\pi} \lambda^{1 / 2}}\right) e^{i k z_{1}}\right] \\
& \left.\quad \times \frac{\epsilon\left(r_{2}, r_{1}\right)\left(\frac{\lambda r_{2}^{3}}{2}+r_{2}^{2}\right) \cos \theta_{12} e^{-\lambda r_{2}}}{r_{2} r_{1}^{2}}\right]
\end{aligned}
$$

where $\lambda=Z / a_{0}$ is the magnitude of the antiproton charge divided by the Bohr radius and the factor of $1 / \sqrt{2}$ is from symmetrizing the two-positron wave function that includes the free positron as (ultimately the $\mathrm{P}$-wave part of [30]) a plane wave.

Temkin [31] justified his use of a step function for the radius at which polarization effects would be included,

$$
\epsilon\left(r_{1}, r_{2}\right)=\left\{\begin{array}{ll}
1, & r_{1}>r_{2} \\
0, & r_{1}<r_{2}
\end{array}\right\},
$$

by noting that "it is not the exact form of the direct polarization potential for small values $r_{1}$ (as long as the potential vanishes in some reasonably fast way as $r_{1} \rightarrow 0$ ) but rather its accuracy for larger values of $r_{1}$ and the consistent incorporation of the exchange polarization, terms which are important for the adiabatic description of scattering." This form in which one uses only dipole polarization has been utilized by a long list of authors for scattering problems.

The counter-argument to including only long-range polarization effects is well laid out by researchers examining of the choice of pseudostates for close-coupling calculations for interactions of electrons with atomic hydrogen. Burke and Schey [32], for instance, note that the convergence using states beyond $n=2$ is very slow, and this "can probably be attributed to the short-range correlation effect, rather than to the long-range polarization." Matese and Oberoi [33] amplify this conclusion: "In the ${ }^{1} S$ channel the $1 s-2 s$ expansion gave a larger correction to the static phase shift than did the $1 s-2 p$ expansion. Since the $2 s$ state contributes nothing to the polarization of the ground state [which is what one would expect from the presence of the cosine function in equation (10) above and in a $2 p$ wave function], the inference is clear."

These researchers do, however, provide some justification for adding polarization effects to improve the finalstate wave function from (5) to (10) in the present case where we are seeking accurate results for very low temperatures that one would associate with very low average energies of the positrons. Burke and Schey [32] note that pseudostates that reproduce $n=1$ polarizabilities should give superior results for very low energies where long-range effects dominate. Matese and Oberoi [33] point out that it is at higher energies (away from resonances) that shortrange effects are expected to dominate.

But even if one were calculating cross sections at somewhat higher energies - and rate coefficients at somewhat higher temperatures - than our focus, more recent formulations extend polarization contributions into the shortrange region. In 2006, Shertzer and Temkin [35] replaced Temkin's cutoff function (11) with a smooth function that allows for polarization at all positive radii, while still going to zero as $r_{1} \rightarrow 0$ :

$\chi_{\mathrm{ST}}\left(r_{1}\right)=1-e^{-2 Z r_{1}}\left(\frac{1}{3} Z^{4} r_{1}^{4}+\frac{4}{3} Z^{3} r_{1}^{3}+2 Z^{2} r_{1}^{2}+2 Z r_{1}+1\right)$.

Bhatia [36] devised an alternative smooth function

$$
\chi_{\mu}\left(r_{1}\right)=\left(1-e^{-\mu r_{1}}\right)^{h}=\sum_{n=0}^{h}\left(\begin{array}{l}
h \\
n
\end{array}\right)(-1)^{n} e^{-n \mu r_{1}}
$$

where $h \geq 3$ and the parameter $\mu$ may be varied to maximize the phase shift (please see Sect. 7.1). Bhatia notes that this term contributes to the short-range correlations in addition to those he obtains from the short-range correlation function that he uses arising from an optical potential.

Table 1 provides values for Bhatia's parameters for three values of $k$. We will utilize Bhatia's form because $\chi_{\mathrm{ST}}$ may be found from it by using just the $h=1$ term in $\chi_{\mu=2 Z}\left(r_{1}\right)$ with $\left(\frac{1}{3}\left(\frac{Z}{2}\right)^{4} \frac{\partial^{4}}{\partial Z^{4}}+\cdots\right)$ multiplying the exponential portion.

Then, using a sum of explicitly correlated functions $e^{-\alpha r_{1}-\beta r_{2}-\gamma r_{12}}$ for the initial state and equation (10) for the final state, the matrix element (4) for ionizing either positron under the influence of the length dipole operator $\left(z_{1}+z_{2}\right)$ is the sum of eight terms:

$$
\begin{aligned}
\mu_{\mathrm{PI}}= & 2\left(I_{11}-P_{11}+I_{21}-P_{21}\right) \frac{\lambda^{3 / 2}}{\sqrt{2 \pi}} \\
= & \iint d^{3} x_{1} d^{3} x_{2} e^{-\alpha r_{1}-\beta r_{2}-\gamma r_{12}}\left(z_{1}+z_{2}\right) \frac{\lambda^{3 / 2}}{\sqrt{\pi}} \frac{1}{\sqrt{2}} \\
& \times\left[e^{-\lambda r_{2}} e^{i k z_{1}}\left(1-\frac{1}{\lambda^{2}} \frac{\chi_{\mu}\left(r_{1}\right)\left(\frac{\lambda r_{2}^{3}}{2}+r_{2}^{2}\right) \cos \theta_{12}}{r_{2} r_{1}^{2}}\right)\right. \\
& \left.+e^{-\lambda r_{1}} e^{i k z_{2}}\left(1-\frac{1}{\lambda^{2}} \frac{\chi_{\mu}\left(r_{2}\right)\left(\frac{\lambda r_{1}^{3}}{2}+r_{1}^{2}\right) \cos \theta_{12}}{r_{1} r_{2}^{2}}\right)\right]
\end{aligned}
$$

where the first factor of two comes from $I_{11}=I_{22}$ and so on, whose subscripts $j$ refer to $z_{j}$ in the dipole operator and in the plane wave, respectively, and we have factored out the coefficient $\frac{\lambda^{3 / 2}}{\sqrt{2 \pi}}$ that is common to all terms.

Keating [16,39] has calculated the non-polarized terms for the more complicated two-electron wave functions of 
Table 1. Parameters for Bhatia's smooth cutoff function at three values of the electron wave number $k$. The exponent $h$ is generally found to be 3 for ${ }^{1} S$ states and 4 for ${ }^{3} S$ states [36].

\begin{tabular}{lllll}
\hline$k$ & $h$ & $\begin{array}{l}\mu \text { (called } \\
\beta \text { in his } \\
\text { papers) }\end{array}$ & $\begin{array}{l}\text { Phase shift } \\
\text { (radians) }\end{array}$ & Source \\
\hline 0 & 3 & 0.54 & & {$[36]$} \\
0.4 & 3 & 0.277 & 0.10047 & {$[37]$} \\
0.8 & 3 & 0.661 & 0.7942772 & {$[38]$} \\
0.8 & 4 & 0.895 & 0.8073684 & {$[38]$} \\
0.8 & 5 & 1.00 & 0.8029370 & {$[38]$} \\
\hline
\end{tabular}

the type

$$
\begin{aligned}
\psi_{H}\left(r_{1}, r_{2}, r_{12}\right)= & \frac{1}{\sqrt{2}}\left(1-\hat{P}_{12}\right) e^{-\alpha r_{1}-\beta r_{2}-\gamma r_{12}} \\
& \times \sum_{l, m, n} c_{l m n} s^{l} t^{2 m} u^{n}
\end{aligned}
$$

where $\hat{P}_{12}$ is the permutation operator for the two identical electrons, with Hylleraas coordinates [40] given by $s=r_{1}+r_{2}, t=r_{1}-r_{2}$, and $u=r_{12} \equiv\left|\mathbf{r}_{1}-\mathbf{r}_{2}\right|$. Equation (11) of Keating et al. [16] with $l=m=n=0$ gives $2\left(I_{11}+I_{21}\right) /\left(8 \pi^{2}\right)$ for the present set of explicitly correlated two-electron wave functions. We find that only the $l=m=n=0$ term requires special care to circumvent the seeming singularity at the origin discussed below, so our results for $P_{21}$ may be extended using Keating's method for use with Hylleraas wave functions.

\section{Reduction of integrals}

The algebra in each case is greatly reduced by making the replacement

$$
e^{-\gamma r_{12}} e^{-r_{2}(\beta+\lambda)}=\left(-\frac{\partial}{\partial \gamma}\right)\left(-\frac{\partial}{\partial \lambda}\right) \frac{e^{-r_{2}(\beta+\lambda)}}{r_{2}} \frac{e^{-\gamma r_{12}}}{r_{12}}
$$

in each non-polarized term, with the notation

$$
I_{j 1}=\left(\frac{\partial}{\partial \gamma}\right)\left(\frac{\partial}{\partial \lambda}\right) R_{0 j 1}
$$

where

$$
R_{011}=\iint d^{3} x_{1} d^{3} x_{2} e^{-\alpha r_{1}} \frac{e^{-r_{2}(\beta+\lambda)}}{r_{2}} \frac{e^{-\gamma r_{12}}}{r_{12}}\left(z_{1} e^{i k z_{1}}\right)
$$

and

$$
R_{021}=\iint d^{3} x_{1} d^{3} x_{2} e^{-\alpha r_{1}} \frac{e^{-r_{2}(\beta+\lambda)}}{r_{2}} \frac{e^{-\gamma r_{12}}}{r_{12}}\left(z_{2} e^{i k z_{1}}\right) .
$$

Likewise, simplifications result from making the replacement

$$
\begin{aligned}
\left(\frac{r_{2} \lambda}{2}+1\right) r_{2}^{2} e^{-\gamma r_{12}} e^{-r_{2}(\beta+\lambda)}= & \left(-\frac{\lambda}{2} \frac{\partial}{\partial \lambda}+1\right)\left(-\frac{\partial}{\partial \gamma}\right) \\
& \times\left(-\frac{\partial}{\partial \lambda}\right) r_{2}^{2} \frac{e^{-r_{2}(\beta+\lambda)}}{r_{2}} \\
& \times \frac{e^{-\gamma r_{12}}}{r_{12}}
\end{aligned}
$$

in each polarized term, with the notation

$$
\begin{aligned}
P_{j 1}= & \frac{1}{\lambda^{2}}\left(-\frac{\lambda}{2} \frac{\partial}{\partial \lambda}+1\right)\left(\frac{\partial}{\partial \gamma}\right)\left(\frac{\partial}{\partial \lambda}\right) \sum_{n=0}^{h} \\
& \times\left(\begin{array}{c}
h \\
n
\end{array}\right)(-1)^{n} \mathcal{R}_{1 n 0 j 1}
\end{aligned}
$$

where the sum over $n$ is from equation (13) and we use a calligraphic font for $\mathcal{R}_{1 \mathrm{n} 0 \mathrm{jk}}$ on the right-hand side to distinguish these reduced forms from the non-polarized reduced forms $R_{0 j k}$ of equation (17). Thus, the polarization integrals to be solved are

$$
\begin{aligned}
\mathcal{R}_{1 n 011}= & \iint d^{3} x_{1} d^{3} x_{2} e^{-(\alpha+n \mu) r_{1}} \frac{e^{-r_{2}(\beta+\lambda)}}{r_{2}} \frac{e^{-\gamma r_{12}}}{r_{12}} \\
& \times\left(\frac{\cos \theta_{12}}{r_{2} r_{1}^{2}}\right)\left(z_{1} e^{i k z_{1}}\right)
\end{aligned}
$$

and

$$
\begin{aligned}
\mathcal{R}_{1 n 021}= & \iint d^{3} x_{1} d^{3} x_{2} e^{-(\alpha+n \mu) r_{1}} \frac{e^{-r_{2}(\beta+\lambda)}}{r_{2}} \frac{e^{-\gamma r_{12}}}{r_{12}} \\
& \times\left(\frac{\cos \theta_{12}}{r_{2} r_{1}^{2}}\right)\left(z_{2} e^{i k z_{1}}\right) .
\end{aligned}
$$

For the cross-terms $\left(z_{2} e^{i k z_{1}}\right)=\left(r_{2} \cos \theta_{2} e^{i k r_{1} \cos \theta_{1}}\right)$, we use the law of cosines,

$$
\cos \theta_{2}=\cos \theta_{1} \cos \theta_{12}+\sin \theta_{1} \sin \theta_{12} \cos \left(\phi_{1}+\phi_{12}\right) .
$$

Ley-Koo and Bunge [41] note that "because the point of interest is on the polar axis" only the cosine-product term contributes, which we confirmed by calculating both terms.

One may expand the plane wave in a series of angular functions and spherical Bessel functions [42], which allows us to do the first angular integral in each of the four terms in equation (14),

$$
\begin{gathered}
\int\left(r_{1} P_{1}\left(\cos \theta_{1}\right)\right)\left(\sum_{l=0}^{\infty}(2 l+1) i^{l} j_{l}\left(k r_{1}\right) P_{l}\left(\cos \theta_{1}\right)\right) d \Omega_{1} \\
=\left(r_{1} \frac{2}{(2+1)} 2 \pi\right)\left((2+1) i^{1} j_{1}\left(k r_{1}\right)\right) .
\end{gathered}
$$

All expressions that follow should in principle be multiplied by this factor of $i$, but since we will take the absolute square of sums of these transition amplitudes to get the cross section, we have ignored this factor.

For all but $P_{21}$, we follow Ley-Koo and Bunge [41] in replacing $d \Omega_{2}$ - the differential solid angle around 
$\hat{r}_{2}$ in a frame of reference in which $\mathbf{r}_{1}$ is taken as the polar axis - by $d \Omega_{12}=\sin \theta_{12} d \theta_{12} d \phi_{12}$. One can immediately integrate over $d \phi_{12}$. They change variables to $\cos \theta_{12}=\left(r_{1}^{2}+r_{2}^{2}-r_{12}^{2}\right) /\left(2 r_{1} r_{2}\right)$ giving $\sin \theta_{12} d \theta_{12}=$ $\left(-2 r_{12}\right) d r_{12} /\left(2 r_{1} r_{2}\right)$, but we instead change variables to $\cos \theta_{12}=u_{12}$ giving $\sin \theta_{12} d \theta_{12}=d u_{12}$.

Aside from providing integrals we have not found in the literature that one needs for the latter method,

$$
\begin{aligned}
& \int_{-1}^{1} d u_{12} \frac{e^{-\gamma \sqrt{r_{1}^{2}-2 r_{1} r_{2} u_{12}+r_{2}^{2}}}}{\sqrt{r_{1}^{2}-2 r_{1} r_{2} u_{12}+r_{2}^{2}}} \\
& =\frac{e^{-\gamma\left|r_{1}-r_{2}\right|}-e^{-\gamma\left|r_{1}+r_{2}\right|}}{\gamma r_{1} r_{2}} \\
& \int_{-1}^{1} d u_{12} \frac{e^{-\gamma \sqrt{r_{1}^{2}-2 r_{1} r_{2} u_{12}+r_{2}^{2}}}}{\sqrt{r_{1}^{2}-2 r_{1} r_{2} u_{12}+r_{2}^{2}}} u_{12} \\
& =\frac{1}{\gamma^{3} r_{1}^{2} r_{2}^{2}}\left(\left(e^{-\gamma\left|r_{1}-r_{2}\right|}+e^{-\gamma\left(r_{1}+r_{2}\right)}\right) r_{1} r_{2} \gamma^{2}\right. \\
& -e^{-\gamma\left|r_{1}-r_{2}\right|}+e^{-\gamma r_{1}-\gamma r_{2}} \\
& \left.+\gamma\left(e^{-\gamma\left(r_{1}+r_{2}\right)}\left(r_{1}+r_{2}\right)-e^{-\gamma\left|r_{1}-r_{2}\right|}\left|r_{1}-r_{2}\right|\right)\right) \\
& \times\left[r_{1}>0, r_{2}>0\right] \\
& \left.\frac{1}{\gamma r_{1} r_{2}} \frac{\partial}{\partial a} \int_{-1}^{1} d u_{12} e^{-\gamma \sqrt{r_{1}^{2}-a 2 r_{1} r_{2} u_{12}+r_{2}^{2}}}\right|_{a=1} u_{12} \\
& =\frac{1}{\gamma^{5} r_{1}^{3} r_{2}^{3}} \frac{\partial}{\partial a} \frac{1}{a^{2}}\left(e^{-\gamma \sqrt{r_{1}^{2}-2 a r_{2} r_{1}+r_{2}^{2}}}\right. \\
& \times\left(-\left(r_{1}^{2}-3 a r_{2} r_{1}+r_{2}^{2}\right) \gamma^{2}\right. \\
& \left.+\mid\left(a \gamma^{2} r_{1} r_{2}-3\right) \sqrt{r_{1}^{2}-2 a r_{2} r_{1}+r_{2}^{2}} \gamma-3\right) \\
& +e^{-\gamma \sqrt{r_{1}^{2}+2 a r_{2} r_{1}+r_{2}^{2}}} \\
& \times\left(\left(r_{1}^{2}+3 a r_{2} r_{1}+r_{2}^{2}\right) \gamma^{2}+\left(a r_{1} r_{2} \gamma^{2}+3\right)\right. \\
& \left.\left.\times \sqrt{r_{1}^{2}+2 a r_{2} r_{1}+r_{2}^{2}} \gamma+3\right)\right)_{a=1},
\end{aligned}
$$

we simply display the results for the non-polarized terms whose derivatives readily give results already found $[16,39]$. These are

$$
\begin{aligned}
R_{011}= & \frac{32 \pi^{2} k}{\gamma^{2}-(\beta+\lambda)^{2}} \\
& \times\left(\frac{1}{\left(k^{2}+(\alpha+\beta+\lambda)^{2}\right)^{2}}-\frac{1}{\left(k^{2}+(\alpha+\gamma)^{2}\right)^{2}}\right)
\end{aligned}
$$

$$
\begin{aligned}
R_{021}= & -\frac{32 \pi^{2}}{\left(\gamma^{2}-(\beta+\lambda)^{2}\right)^{2}}\left(\frac{k\left((\beta+\lambda)^{2}-\gamma^{2}\right)}{\left(k^{2}+(\alpha+\beta+\lambda)^{2}\right)^{2}}\right. \\
& +\frac{\gamma(\alpha+\gamma)}{k\left(k^{2}+(\alpha+\gamma)^{2}\right)}-\frac{(\beta+\lambda)(\alpha+\beta+\lambda)}{k\left(k^{2}+(\alpha+\beta+\lambda)^{2}\right)} \\
& \left.-\frac{\alpha \tan ^{-1}\left(\frac{k}{\alpha+\beta+\lambda}\right)}{k^{2}}+\frac{\alpha \tan ^{-1}\left(\frac{k}{\alpha+\gamma}\right)}{k^{2}}\right) \cdot
\end{aligned}
$$

Then the final contribution these two make to the matrix element (14) is

$$
\begin{aligned}
I_{11}+I_{21}= & \iint d^{3} x_{1} d^{3} x_{2} e^{-\alpha r_{1}-\beta r_{2}-\gamma r_{12}} \\
& \times\left(z_{1}+z_{2}\right) e^{-\lambda r_{2}} e^{i k z_{1}} \\
= & \left(\frac{\partial}{\partial \gamma}\right)\left(\frac{\partial}{\partial \lambda}\right)\left(R_{011}+R_{021}\right),
\end{aligned}
$$

whose final form after the derivatives [16] are taken we will not repeat here because of its complexity.

In anticipation of its usefulness for amplitude integrals beyond the dipole approximation that involve even higher powers of $\cos \theta_{12}$, we introduce an addition theorem from Magnus et al. [43]

$$
\begin{aligned}
& \frac{\exp \left(-\eta \sqrt{x_{1}^{2}-2 \mathbf{x}_{1} \cdot \mathbf{x}_{2}+x_{2}^{2}}\right)}{\sqrt{x_{1}^{2}-2 \mathbf{x}_{1} \cdot \mathbf{x}_{2}+x_{2}^{2}}} \\
& \quad=x_{1}^{-1 / 2} x_{2}^{-1 / 2} \sum_{n=0}^{\infty}(2 n+1) P_{n}(\cos \theta) \\
& \quad \times I_{n+1 / 2}\left(\eta x_{1}\right) K_{n+1 / 2}\left(\eta x_{2}\right) \quad\left[0<x_{1}<x_{2}\right]
\end{aligned}
$$

that can be recast into modern notion,

$$
\begin{aligned}
& \frac{\exp \left(-\eta \sqrt{x_{1}^{2}-2 \mathbf{x}_{1} \cdot \mathbf{x}_{2}+x_{2}^{2}}\right)}{\sqrt{x_{1}^{2}-2 \mathbf{x}_{1} \cdot \mathbf{x}_{2}+x_{2}^{2}}} \\
& =x_{1}^{-1 / 2} x_{2}^{-1 / 2} \sum_{n=0}^{\infty}(2 n+1) P_{n}(\cos \theta) \\
& \quad \times I_{n+1 / 2}\left(\eta x_{<}\right) K_{n+1 / 2}\left(\eta x_{>}\right),
\end{aligned}
$$

whose integral with powers of $\cos \theta$ (recast as sums of Legendre Polynomials [44,45])

$$
2 \pi \int_{-1}^{1} d u P_{L}(u) P_{n}(u)=2 \pi \frac{2}{2 L+1} \delta_{L n}
$$

will project out a sum of several terms. Then for the direct polarization term,

See equation (31) next page.

and 


$$
\begin{aligned}
& \mathcal{R}_{1 n 011} \\
& =4 \pi \int_{0}^{\infty} d r_{1} r_{1} j_{1}\left(k r_{1}\right) e^{-r_{1}(\alpha+n \mu)} \\
& \times\left(\int_{0}^{r_{1}} d r_{2} \frac{2 \pi\left(\frac{1}{r_{1} \gamma}+1\right) e^{-r_{2}(\beta+\lambda)-r_{1} \gamma}\left(2 \cosh \left(r_{2} \gamma\right)-\frac{2 \sinh \left(r_{2} \gamma\right)}{r_{2} \gamma}\right)}{\gamma r_{1}}\right. \\
& \left.+\int_{r_{1}}^{\infty} d r_{2} \frac{2 \pi\left(\frac{1}{r_{2} \gamma}+1\right) e^{-r_{2}(\beta+\lambda)-r_{2} \gamma}\left(2 \cosh \left(r_{1} \gamma\right)-\frac{2 \sinh \left(r_{1} \gamma\right)}{r_{1} \gamma}\right)}{\gamma r_{1}}\right) \\
& =\frac{16 \pi^{2}}{k^{2}\left((\beta+\lambda)^{2}-\gamma^{2}\right)^{2}}\left(k(\beta-\gamma+\lambda)-\frac{k\left((\beta+\lambda)^{2}-\gamma^{2}\right)(n \mu+\alpha+\beta+\lambda)}{k^{2}+(n \mu+\alpha+\beta+\lambda)^{2}}\right. \\
& \left.+\left(k^{2}+(n \mu+\alpha)^{2}-\gamma^{2}\right)\left(\tan ^{-1}\left(\frac{k}{n \mu+\alpha+\beta+\lambda}\right)-\tan ^{-1}\left(\frac{k}{n \mu+\alpha+\gamma}\right)\right)\right) .
\end{aligned}
$$

$$
\begin{aligned}
& \mathcal{R}_{1 n 021} \\
& =\frac{4^{2} \pi^{2}}{\left((\beta+\lambda)^{2}-\gamma^{2}\right)^{3}} \int_{0}^{\infty} d r_{1} j_{1}\left(k r_{1}\right) e^{-r_{1}(\alpha+n \mu)}\left(\frac{2 e^{-\gamma r_{1}}\left(\left((\beta+\lambda)^{2}+3 \gamma^{2}\right)\right)}{r_{1}}\right. \\
& +\frac{16 e^{-\gamma r_{1}}}{r_{1}^{3}}+\frac{16 \gamma e^{-\gamma r_{1}}}{r_{1}^{2}}-\frac{16 e^{-(\beta+\lambda) r_{1}}}{r_{1}^{3}}-\frac{16(\beta+\lambda) e^{-(\beta+\lambda) r_{1}}}{r_{1}^{2}} \\
& \left.-\frac{e^{-(\beta+\lambda) r_{1}}\left(r_{1}^{2}\left((\beta+\lambda)^{2}-\gamma^{2}\right)^{2}+4 r_{1}\left((\beta+\lambda)^{3}-\gamma^{2}(\beta+\lambda)\right)+2\left(5(\beta+\lambda)^{2}-\gamma^{2}\right)\right)}{r_{1}}\right) .
\end{aligned}
$$

The polarization cross-term $\mathcal{R}_{1 n 021}$, however, runs afoul of unresolvable singularities in the $r_{1}$ integral using this conventional approach.

\section{Bypassing singularities in $\mathcal{R}_{1 n 021}$}

A similar approach gives

See equation (32) above.

Consider the portion of this integral containing terms that are singular when taken alone, and thus cannot be integrated analytically term-by-term,

$$
\begin{aligned}
S= & \frac{4^{2} \pi^{2}}{\left((\beta+\lambda)^{2}-\gamma^{2}\right)^{3}} \int_{0}^{\epsilon} d r_{1}\left(j_{1}\left(k r_{1}\right) e^{-(n \mu+\alpha) r_{1}}\right. \\
& \times\left[\frac{16 e^{-\gamma r_{1}}-16 e^{-(\beta+\lambda) r_{1}}}{r_{1}^{3}}\right. \\
& \left.\left.+\frac{16 \gamma e^{-\gamma r_{1}}-16(\beta+\lambda) e^{-(\beta+\lambda) r_{1}}}{r_{1}^{2}}\right]\right) .
\end{aligned}
$$

If we expand the exponentials in the $r_{1}^{-3}$ term of (33) in a Taylor series, one sees that the constant term is selfcanceling. The second term gives $\frac{-16 \gamma+16(\beta+\lambda)}{r_{1}^{2}}$, which precisely cancels the first term of a Taylor series expansion of the exponentials in the $r_{1}^{-2}$ term of (33). Differing factorials forestall precise cancellation of the remaining pairs of terms, but fortunately the spherical Bessel function has behavior near the origin [42]

$$
j_{1}(z) \sim \frac{\sqrt{\pi} 2^{-2} z^{1}}{\Gamma\left(1+\frac{3}{2}\right)} .
$$

This multiplies the $r_{1}^{-1}$ terms in the two series, and the resulting integral is a finite constant times $\epsilon$, which may be made a small as we like. The next pair of terms integrate to $\epsilon^{2}$, which go to zero more rapidly than the $r_{1}^{-1}$ terms, and so on. Thus the individually singular portion of the integral when taken as a pair gives zero contribution.

That does not, however, solve our problem in full. One may show numerically that the manifestly nonsingular portion of the infinite integral of those same terms

$$
\begin{aligned}
\mathrm{NS}= & \frac{4^{2} \pi^{2}}{\left((\beta+\lambda)^{2}-\gamma^{2}\right)^{3}} \int_{\epsilon}^{\infty} d r_{1}\left(j_{1}\left(k r_{1}\right) e^{-(n \mu+\alpha) r_{1}}\right) \\
& \times\left[\frac{16 e^{-\gamma r_{1}}-16 e^{-(\beta+\lambda) r_{1}}}{r_{1}^{3}}\right. \\
& \left.+\frac{16 \gamma e^{-\gamma r_{1}}-16(\beta+\lambda) e^{-(\beta+\lambda) r_{1}}}{r_{1}^{2}}\right]
\end{aligned}
$$


approaches a stable value as $\epsilon$ becomes small but nonzero, but this integral nevertheless defies analytic integration. The $r_{1}^{-1}$ terms in (32) are easily integrated, but that is not useful without an analytically result for (35), above.

\section{An integro-differential transform}

One must instead turn to alternative methods of integration to have any hope of a fully analytical solution. The Gaussian transform is a sufficiently different approach to have some promise of avoiding the above problems. Its purpose is to move the multi-center coordinates into a quadratic form in an exponential so that one may complete the square and thereby perform the spatial integral(s). In 1990 one of us [46] extended the Gaussian transform to an integro-differential transform allowing one to also move the angular variables within spherical harmonics of non-spherically-symmetric wave functions into that quadratic form. Since our dipole operator and the angular function in the polarization term are both spherical harmonics $\left(\cos \theta=2 \sqrt{\frac{\pi}{3}} \mathrm{Y}_{1,0}(\theta, \phi)\right)$, we can use that formalism. We find it convenient to modify this formalism slightly by setting $\cos \theta_{12}=-\left.x_{1}^{-1} x_{2}^{-1} \frac{\partial}{\partial Q}\right|_{Q=0} e^{-Q \mathbf{x}_{1} \cdot \mathbf{x}_{2}}$ and applying this to the two-center integral over $x_{2}$. Thus, we need to calculate

$$
\begin{aligned}
\mathcal{R}_{1 n 021}= & 8 \pi^{2} \int_{0}^{\infty} d r_{1} r_{1}^{2} j_{1}\left(k r_{1}\right) e^{-r_{1}(\alpha+n \mu)} \frac{1}{r_{1}^{2}} S_{Q 1}^{\beta+\lambda 0 \gamma 0} \\
& \times\left(0 ; 0, r_{1}\right)
\end{aligned}
$$

where

$$
\begin{aligned}
& S_{Q 1}^{\eta_{1} 0 \eta_{2} 0}\left(0 ; 0, x_{1}\right) \\
& \quad=\int d^{3} x_{2}\left(x_{2} \cos \theta_{12}\right)^{2} \frac{e^{-\eta_{1} x_{2}}}{x_{2}} \frac{e^{-\eta_{2} x_{12}}}{x_{12}} \\
& \quad=\left.x_{1}^{-2} \frac{\partial^{2}}{\partial Q^{2}}\right|_{Q=0} \int d^{3} x_{2} x_{2}^{2-2} e^{-Q \mathbf{r}_{1} \cdot \mathbf{r}_{2}} \frac{e^{-\eta_{1} x_{2}}}{x_{2}} \frac{e^{-\eta_{2} x_{12}}}{x_{12}} \\
& \quad=\left.x_{1}^{-2} \frac{\partial^{2}}{\partial Q^{2}}\right|_{Q=0} S_{1}^{\eta_{1} 0 \eta_{2} 0}\left(Q \mathbf{r}_{1} / i ; 0, x_{1}\right)
\end{aligned}
$$

and the superscripts and subscripts are in the notation of prior work [46].

We introduce the Gaussian transforms of the two Yukawa potentials (see the terms in square brackets in the first line in the equation below), complete the square, change variables to $\mathbf{x}_{2}^{\prime}=\mathbf{x}_{2}-\frac{\mathbf{x}_{1} \rho_{2}-\frac{i \mathbf{p}}{2}}{\rho_{1}+\rho_{2}}$ with unit Jacobian, perform the $\mathbf{x}_{2}^{\prime}$ integral [47], transform from the Gaussian integration variable $\rho_{1}$ to $\tau_{1}=\frac{\rho_{1}}{\rho_{1}+\rho_{2}}$, and do the $\rho_{2}$ integral [48]. These steps give

$$
\begin{aligned}
& S_{1}^{\eta_{1} 0 \eta_{2} 0}\left(\mathbf{p} ; 0, x_{1}\right) \\
& =\int d^{3} x_{2}\left[\frac{1}{\sqrt{\pi}} \int_{0}^{\infty} d \rho_{1} \frac{e^{-x_{2}^{2} \rho_{1}} e^{-\eta_{1}^{2} / 4 / \rho_{1}}}{\rho_{1}^{1 / 2}}\right] \\
& \times\left[\frac{1}{\sqrt{\pi}} \int_{0}^{\infty} d \rho_{2} \frac{e^{-x_{12}^{2} \rho_{2}} e^{-\eta_{2}^{2} / 4 / \rho_{2}}}{\rho_{2}{ }^{1 / 2}}\right] e^{-i \mathbf{p} \cdot \mathbf{x}_{2}} \\
& =\frac{1}{\pi} \int d^{3} x_{2} \int_{0}^{\infty} d \rho_{1} \frac{e^{-\eta_{1}^{2} / 4 / \rho_{1}}}{\rho_{1}^{1 / 2}} \int_{0}^{\infty} d \rho_{2} \frac{e^{-\eta_{2}^{2} / 4 / \rho_{2}}}{\rho_{2}^{1 / 2}} \\
& \times \exp \left(-\left(\rho_{1}+\rho_{2}\right)\left(x_{2}-\frac{x_{1} \rho_{2}-\frac{i p}{2}}{\rho_{1}+\rho_{2}}\right)^{2}\right. \\
& \left.+\frac{\left(x_{1} \rho_{2}-\frac{i p}{2}\right)^{2}}{\rho_{1}+\rho_{2}}-\rho_{2} x_{1}^{2}\right) \\
& =\pi^{1 / 2} \int_{0}^{\infty} d \rho_{1} \frac{e^{-\eta_{1}^{2} / 4 / \rho_{1}}}{\rho_{1}^{1 / 2}} \int_{0}^{\infty} d \rho_{2} \frac{e^{-\eta_{2}^{2} / 4 / \rho_{2}}}{\rho_{2}{ }^{1 / 2}} \\
& \times \frac{1}{\left(\rho_{1}+\rho_{2}\right)^{3 / 2}} \\
& \times \exp \left(-\frac{x_{1}^{2} \rho_{1} \rho_{2}}{\rho_{1}+\rho_{2}}-\frac{i \mathbf{p} \cdot \mathbf{x}_{1} \rho_{2}}{\rho_{1}+\rho_{2}}-\frac{\mathrm{p}^{2}}{4\left(\rho_{1}+\rho_{2}\right)}\right) \\
& =\pi^{1 / 2} \int_{0}^{1} d \tau_{1} \frac{1}{\tau_{1}^{1 / 2}\left(1-\tau_{1}\right)^{3 / 2}} \\
& \times \int_{0}^{\infty} d \rho_{2} e^{-\eta_{2}^{2} / 4 / \rho_{2}} e^{-\eta_{1}^{2}\left(1-\tau_{1}\right) / \tau_{1} / 4 / \rho_{2}} \\
& \times \frac{\left(1-\tau_{1}\right)^{3 / 2}}{\rho_{2}^{3 / 2}} \exp \left(-x_{1}^{2} \rho_{2} \tau_{1}-i \mathbf{p} \cdot \mathbf{x}_{1}\left(1-\tau_{1}\right)\right. \\
& \left.-\frac{\mathrm{p}^{2}}{4} \frac{\left(1-\tau_{1}\right)}{\rho_{2}}\right) \\
& =2 \pi \int_{0}^{1} d \tau_{1} e^{-i \mathbf{p} \cdot \mathbf{x}_{1}\left(1-\tau_{1}\right)} \frac{\exp \left(-x_{1} L^{\prime}\right)}{L^{\prime}}
\end{aligned}
$$

where

$$
L^{\prime}=\sqrt{\left(\tau_{1} \mathrm{p}^{2}+\eta_{1}^{2}\right)\left(1-\tau_{1}\right)+\tau_{1} \eta_{2}^{2}}
$$

Cheshire [49] reduced the related integral (his Eq. (19))

$$
\begin{aligned}
I_{1}= & \left.\frac{\eta_{1}^{3 / 2}}{\sqrt{\pi}} S_{1 s 1}^{\eta_{1} 0 \eta_{2} 0}\left(\frac{1}{2} \mathbf{k}_{\mathbf{f}} ; 0, x_{2}\right)\right|_{\eta_{1}=1, \eta_{2}=1 / 2} \\
= & \int d^{3} x_{1} \frac{\eta_{2}^{3 / 2}}{\sqrt{\pi}} e^{-\eta_{2} x_{12}} \frac{\eta_{1}^{3 / 2}}{\sqrt{\pi}} \frac{e^{-\eta_{1} x_{1}}}{x_{1}} \\
& \times\left. e^{-i \frac{1}{2} \mathbf{k}_{\mathbf{f}} \cdot \mathbf{x}_{1}}\right|_{\eta_{1}=1, \eta_{2}=1 / 2} \\
= & \frac{\eta_{1}^{3 / 2}}{\sqrt{\pi}} \frac{\eta_{2}^{3 / 2}}{\sqrt{\pi}}\left(-\frac{\partial}{\partial \eta_{2}}\right) \int d^{3} x_{1} \frac{e^{-\eta_{2} x_{12}}}{x_{12}} \frac{e^{-\eta_{1} x_{1}}}{x_{1}} \\
& \times\left. e^{-i \frac{1}{2} \mathbf{k}_{\mathbf{f}} \cdot \mathbf{x}_{1}}\right|_{\eta_{1}=1, \eta_{2}=1 / 2} \\
= & \frac{\eta_{1}^{3 / 2}}{\sqrt{\pi}} \frac{\eta_{2}^{3 / 2}}{\sqrt{\pi}}\left(-\frac{\partial}{\partial \eta_{2}}\right) \\
& \times\left. S_{1}^{\eta_{1} 0 \eta_{2} 0}\left(\frac{1}{2} \mathbf{k}_{\mathbf{f}}, ; 0, x_{2}\right)\right|_{\eta_{1}=1, \eta_{2}=1 / 2},
\end{aligned}
$$

which matches the present result after substituting the specialized values for $\eta_{1}$ and $\eta_{2}$. Unlike Cheshire's application, we are able to integrate this one-dimensional integral because we set the momentum term to $Q=0$ after taking the $Q$ derivatives. For this integration, it is helpful to change variables to $s=\left[\left(\eta_{2}^{2}-\eta_{1}^{2}\right) \tau_{1}+\eta_{1}^{2}\right]^{1 / 2}$ : 


$$
\begin{aligned}
& \mathcal{R}_{1 n 021} \\
& =-16 \pi^{2} \int_{\beta+\lambda}^{\gamma} d s \frac{\left(s^{2}-\gamma^{2}\right)}{k^{2} s^{2}\left((\beta+\lambda)^{2}-\gamma^{2}\right)^{3}}\left(\frac{2 k^{3} s^{2}\left(s^{2}-\gamma^{2}\right)}{\left(k^{2}+(n \mu+s+\alpha)^{2}\right)^{2}}\right. \\
& +\left((\beta+\lambda)^{2}-s^{2}\right)\left(k-(n \mu+s+\alpha) \tan ^{-1}\left(\frac{k}{n \mu+s+\alpha}\right)\right) \\
& \left.+\frac{s\left((\beta+\lambda)^{2}-s^{2}\right)\left(\left(k^{2}+(n \mu+s+\alpha)^{2}\right) \tan ^{-1}\left(\frac{k}{n \mu+s+\alpha}\right)-k(n \mu+s+\alpha)\right)}{k^{2}+(n \mu+s+\alpha)^{2}}\right) \\
& =\frac{16 \pi^{2}}{3 k^{2}\left((\beta+\lambda)^{2}-\gamma^{2}\right)^{3}}\left[k(\alpha+3 \mu)(\alpha+\gamma+3 \mu)^{2}+3 k \gamma^{2}(\beta+\lambda)-3 k \gamma(\beta+\lambda)^{2}\right. \\
& -3 k\left(k^{2}+(\alpha+5 \mu)^{2}\right)(\alpha+\gamma+5 \mu)-k(\alpha+4 \mu)(\alpha+\beta+\lambda+4 \mu)^{2} \\
& +3 k\left(k^{2}+(\alpha+6 \mu)^{2}\right)(\alpha+\beta+\lambda+6 \mu)+3 k\left(k^{2}(3 \alpha-\gamma+21 \mu)\right. \\
& \left.-(\alpha-\gamma+7 \mu)^{2}(\alpha+\gamma+7 \mu)\right)+\frac{3 k}{k^{2}+(n \mu+\alpha+\beta+\lambda)^{2}} \\
& \times\left(k^{4}(-3(n \mu+\alpha)+\beta+\lambda)+\left(n^{2} \mu^{2}+2 n \alpha \mu+\alpha^{2}-\gamma^{2}\right)^{2}(n \mu+\alpha+\beta+\lambda)\right. \\
& -2 k^{2}\left((\beta+\lambda)\left(3 n^{2} \mu^{2}+6 n \alpha \mu+3 \alpha^{2}-\gamma^{2}\right)+(n \mu+\alpha)\right. \\
& \left.\left.\times\left(n^{2} \mu^{2}+2 n \alpha \mu+\alpha^{2}+\gamma^{2}\right)\right)\right)-(n \mu+\alpha) \tan ^{-1}\left(\frac{k}{n \mu+\alpha+\gamma}\right) \\
& \times\left(-3 \gamma\left(\beta^{2}+2 \beta \lambda+\gamma^{2}+\lambda^{2}\right)-3 \gamma(\beta+\lambda)^{2}+\gamma^{3}\right. \\
& \left.+(n \mu+\alpha)\left(n^{2} \mu^{2}+2 n \alpha \mu+\alpha^{2}-3 \beta^{2}-6 \beta \lambda-3 \gamma^{2}-3 \lambda^{2}\right)\right) \\
& -3 \tan ^{-1}\left(\frac{n \mu+\alpha+\gamma}{k}\right)\left(k^{2}\left(4 n^{2} \mu^{2}+8 n \alpha \mu+4 \alpha^{2}+\beta^{2}+2 \beta \lambda-\gamma^{2}+\lambda^{2}\right)\right. \\
& +4 \alpha\left(n^{3} \mu^{3}-n \gamma^{2} \mu\right)-2 k^{4}+n^{4} \mu^{4}-2 \alpha^{2}\left(\gamma^{2}-3 n^{2} \mu^{2}\right) \\
& \left.-2 n^{2} \gamma^{2} \mu^{2}+4 n \alpha^{3} \mu+\alpha^{4}+\beta^{2} \gamma^{2}+2 \beta \gamma^{2} \lambda+\gamma^{4}+\gamma^{2} \lambda^{2}\right) \\
& +(n \mu+\alpha) \tan ^{-1}\left(\frac{k}{n \mu+\alpha+\beta+\lambda}\right)\left(-3(\beta+\lambda)\left(\beta^{2}+2 \beta \lambda+\gamma^{2}+\lambda^{2}\right)\right. \\
& -3 \gamma^{2}(\beta+\lambda)+(\beta+\lambda)^{3} \\
& \left.+(n \mu+\alpha)\left(n^{2} \mu^{2}+2 n \alpha \mu+\alpha^{2}-3 \beta^{2}-6 \beta \lambda-3 \gamma^{2}-3 \lambda^{2}\right)\right) \\
& +3 \tan ^{-1}\left(\frac{n \mu+\alpha+\beta+\lambda}{k}\right)\left(-2 k^{4}+\alpha^{4}+\beta^{2} \gamma^{2}+2 \beta \gamma^{2} \lambda+\gamma^{4}+\gamma^{2} \lambda^{2}\right. \\
& +n^{4} \mu^{4}-2 \alpha^{2}\left(\gamma^{2}-3 n^{2} \mu^{2}\right)-2 n^{2} \gamma^{2} \mu^{2}+4 n \alpha^{3} \mu \\
& \left.+k^{2}\left(4 n^{2} \mu^{2}+8 n \alpha \mu+4 \alpha^{2}+\beta^{2}+2 \beta \lambda-\gamma^{2}+\lambda^{2}\right)+4 \alpha\left(n^{3} \mu^{3}-n \gamma^{2} \mu\right)\right) \\
& +8 k^{3}(n \mu+\alpha) \log \left(k^{2}+(n \mu+\alpha+\gamma)^{2}\right) \\
& \left.-8 k^{3}(n \mu+\alpha) \log \left(k^{2}+(n \mu+\alpha+\beta+\lambda)^{2}\right)\right] \text {. }
\end{aligned}
$$

$$
\begin{aligned}
S_{Q 1}^{\eta_{1} 0 \eta_{2} 0}\left(0 ; 0, x_{1}\right) & \left.\right|_{Q=0} \\
= & 2 \pi \int_{0}^{1} d \tau_{1} \frac{\left(1-\tau_{1}\right) e^{-x_{1}} \sqrt{\left(\eta_{2}^{2}-\eta_{1}^{2}\right) \tau_{1}+\eta_{1}^{2}}}{\left(\left(\eta_{2}^{2}-\eta_{1}^{2}\right) \tau_{1}+\eta_{1}^{2}\right)^{3 / 2}} \\
& \times x_{1}^{2}\left(\tau_{1}-1\right)\left(\eta_{1}^{2}\left(\tau_{1}-1\right)-\eta_{2}^{2} \tau_{1}\right) \\
& +x_{1} \tau_{1} \sqrt{\left(\eta_{2}^{2}-\eta_{1}^{2}\right) \tau_{1}+\eta_{1}^{2}}+\tau_{1} \\
= & 4 \pi \int_{\eta_{1}}^{\eta_{2}} d s \frac{e^{-s x_{1}\left(s^{2}-\eta_{2}^{2}\right)\left(x_{1}\left(s \eta_{1}^{2}-s^{3}\right)-s^{2}+x_{1}^{2}\left(s^{4}-s^{2} \eta_{2}^{2}\right)+\eta_{1}^{2}\right)}}{s^{2}\left(\eta_{2}^{2}-\eta_{1}^{2}\right)^{3}} .
\end{aligned}
$$

The next step would normally be integration over $s$ followed by $x_{1}$, but that would land us back in singularity trouble. If we instead first integrate over $x_{1}$ and then over $s$, we obtain a nonsingular analytical result:

See equation (42) above.

The results of the remaining steps of taking the derivatives of all four terms indicated in equations (16) and (19) are quite long and will not be displayed.

\section{The cutoff function}

The above expression contains the parameter $\mu$ from Bhatia's [36] smooth cutoff function, equation (13), which may 
be varied to maximize the $e^{ \pm}-H$ scattering phase shift. Let us take a moment to outline what that entails.

\subsection{Scattering formalism}

The reason so many workers have utilized Chandrasekhar's [24,25] formulation of the cross section for photoionization, $\sigma_{\mathrm{PI}}$, is that (whether or not it could somehow be derived from a scattering $S$-matrix) equation (2) has the form of a bound-state transition amplitude with one of the atomic states simply lying above the ionization threshold as in some pseudostate formulations. (It is for this very reason that the radiative attachment cross section can be most easily found from the photoionization cross section via the principle of detailed balance (see, e.g., [21]) as opposed to starting initially with radiative attachment, which requires a fully-formed scattering formulation.) For instance, in the parallel $\mathrm{Ps}^{-}$case, Ward, McDowell, and Humberston [30] describe this formulation as calculating an allowed dipole transition to the continuum of the two-electron Hamiltonian.

Chandrasekhar's formulation, at its simplest, has the photoionized electron represented by a plane wave, multiplying a hydrogen ground state wave function as in equation (5), whose behavior at infinite distance is ignored, and ultimately whose $\mathrm{P}$-wave part [30] is all that is used. Conventional scattering formulations of a transition amplitude, on the other hand, have a final state for the outgoing positron that is represented by the product of a scattering function $u$ and a hydrogen ground state wave function,

$$
\psi_{f s}=\frac{1}{\sqrt{2 \pi}}\left(u\left(\mathbf{r}_{1}\right) e^{-r_{2}}+u\left(\mathbf{r}_{12}\right) e^{-r_{1}}\right),
$$

where at large distances scattering function $u$ is the superposition of a plane wave and an outgoing spherical wave. The form taken by a model potential is [50-52]

$$
u(\mathbf{r}) \underset{r \rightarrow \infty}{\longrightarrow} A\left(\exp (i \mathbf{k} \cdot \mathbf{r})+f(\theta, \phi) \frac{\exp (i k r)}{r}\right) .
$$

Such a scattering formulation does not find the cross section from an equation like (2), but instead the differential cross section is the absolute square of the scattering amplitude $f$ of (44).

For a case like the present one, the problem is usually given in a partial wave expansion of the plane wave, as in the inner parentheses of equation (23), in which the outgoing boundary condition may be expressed as [53-55]

$$
u(\mathbf{r}) \underset{r \rightarrow \infty}{\longrightarrow} A_{l}(k) \sin \left(k r-\frac{1}{2} l \pi+\delta_{l}(k)\right) .
$$

The last term $\delta_{l}(k)$ is called the phase shift, and for $l=0$ it is called the s-wave phase shift, and so on. The scattering amplitude is then given by [56-58]

$$
f(\theta, \phi)=\frac{1}{2 i k} \sum_{l=0}^{\infty}(2 l+1)\left[\exp \left(2 i \delta_{l}(k)\right)-1\right] P_{l}(\cos \theta) .
$$

Bhatia [20] includes short-range correlations using the projection-operator (optical potential) formalism of Feshbach [59] and he notes [60] that the phase shifts found by this method are lower bounds to the exact phase shifts, unlike those derived from Kohn variational methods. Therefore, Bhatia is able to vary the wave function representing short-range correlations, along with the polarization parameter $\mu$, in order to maximize the phase shift to make it as exact as possible.

\subsection{A workaround}

Unfortunately for those of us without code at hand to calculate the phase shift, this presents a problem. One solution is to use a polynomial approximation. We fit $\mu$ to Bhatia's data points $\mu=0.54$ at $k=0[36], \mu=0.277$ at $k=0.4$ [37], and $\mu=0.661$ for $k=0.8$ [38], all with $h=3: \mu(k)=0.54-1.46625 k+2.021875 k^{2}$. One may also use Shertzer and Temkin's [35] smooth cutoff function within the present formalism by taking a single exponential term with $h=1$ and $\mu=2 Z$ and taking derivatives of $Z$ to generate the powers of $r_{1}$ in (12). This significantly increases the algebraic complexity of the results, and hence the time required for the calculations, but poses no other problem. We found that the photodetachment results were not greatly different from Bhatia's [20] polarization results, except at the highest energies (see the fifth column in Tab. 2). Note that Bhatia also calculated cross sections with short-range correlations arising from an optical potential as well as the long-range correlations that arise from polarization (his Tab. IV). These are included in column 6 , and markedly increase the cross section at highest energies but have little effect below $k=0.6$.

This reasonably good match of our results (solid line) with Bhatia's [20] results (stars) is also seen in Figure 1, except at the smallest wavelengths.

One sees in Figure 1b a moderate shift downward in our present results (solid black) using a three-term polynomial approximation to Bhatia's smooth cutoff function parameter $\mu$ from our unpolarized previous work (gray dashed) [16]. Use of the cutoff function of Shertzer and Temkin [35] (the gray dot-dot-dashed curve) doubles this downward shift at peak. Bhatia's polarized results are the stars at graph coordinates $(7.7,3.91)$ and $(8.4,3.96)$. Figure $1 \mathrm{c}$ shows the sharp upward bend of Bhatia's results at small wavelengths (high energies), also seen in the last three rows of Table 2 .

Let us compare our results to a representative subset of the additional ways the cross section for photoionizing an electron from the $\left(1 s^{2}{ }^{1} S^{e}\right)$ state of the negative hydrogen ion $\mathrm{H}^{-}$has been calculated, encompassing a wide variety of initial and the final state wave functions:

(i) Venuti and Decleva [63] (small dashed) use a close coupling (CC) approach extended to include correlating configurations in the final state and a configuration interaction (CI) initial state extended by inclusion of B-spline bases.

(ii) Saha [64] (medium dashed) uses multiconfiguration Hartree-Fock (MCHF) wave functions for both the final and the initial states. 
Table 2. The cross section for photoionizing an electron from the $\left(1 s^{2}{ }^{1} S^{e}\right)$ state of the negative hydrogen ion $\mathrm{H}^{-}$in units of $10^{-17} \mathrm{~cm}^{2}$ as a function of outgoing electron wave number, $k$. Column 2 is our prior unpolarized result [16] and column 3 is the result of our three-term polynomial approximation to Bhatia's smooth cutoff function parameter $\mu(k)=0.54-1.46625 k+$ $2.021875 k^{2}$. Both were calculated using a 200-term wave function [18] composed of explicitly correlated exponentials, as was fourth column using the cutoff function of Shertzer and Temkin [35]. Bhatia's [20] results using a 364-term Hylleraas wave function and his cutoff function with the parameter $\mu$ varied for each $k$ to maximize the phase shift are given in column 5 for only long-range polarization effects. Column 6 adds in his short-range correlations arising from an optical potential. The remaining columns encompass the work of Venuti and Decleva [63], Saha [64], Wishart [65], Stewart [66], Broad and Reinhardt, and Ajmera and Chung [68].

\begin{tabular}{|c|c|c|c|c|c|c|c|c|c|c|c|c|}
\hline$k$ & Unpolarized & $\begin{array}{l}\text { Present } \\
\text { work } \\
\text { Poly. } \\
\text { approx. to } \\
\mu\end{array}$ & $\mathrm{ST}$ & $\begin{array}{l}\text { Bhatia } \\
\text { (Tab. III) } \\
\text { polarization } \\
\text { only }\end{array}$ & $\begin{array}{l}\text { Bhatia } \\
\text { (Tab. IV) } \\
\text { w/short- } \\
\text { range } \\
\text { corr. }\end{array}$ & $\begin{array}{l}\text { Venuti and } \\
\text { Decleva }\end{array}$ & Saha & Wishart & Stewart & $\begin{array}{l}\text { Broad and } \\
\text { Reinhardt }\end{array}$ & $\begin{array}{l}\text { Ajmera and } \\
\text { Chung }\end{array}$ & $10^{3} \AA$ \\
\hline 0.01 & 0.00281 & 0.00245 & 0.00245 & 0.00245 & & & & & & & & 16.4 \\
\hline 0.02 & 0.01973 & 0.01927 & 0.01924 & 0.01959 & & & & & & & & 16.3 \\
\hline 0.03 & 0.06433 & 0.06332 & 0.06324 & 0.06444 & & & & & & & & 16.2 \\
\hline 0.04 & 0.14629 & 0.14464 & 0.14443 & 0.14736 & 0.1475 & & & & & & & 16 \\
\hline 0.05 & 0.27124 & 0.26957 & 0.26916 & 0.2748 & 0.27517 & 0.2749 & 0.284 & & & & & 15.7 \\
\hline 0.06 & 0.44174 & 0.44029 & 0.43956 & 0.44914 & 0.44988 & & & & & & & 15.4 \\
\hline 0.07 & 0.65834 & 0.65495 & 0.65374 & 0.66844 & & & & & & & & 15.1 \\
\hline 0.1 & 1.50045 & 1.49507 & 1.49225 & 1.52465 & 1.53024 & 1.5314 & 1.554 & & & & & 13.9 \\
\hline 0.2 & 3.83254 & 3.81076 & 3.78552 & 3.83688 & 3.85443 & 3.8568 & 3.911 & 3.898 & 3.912 & 3.982 & 3.8625 & 9.5 \\
\hline 0.23 & 3.96543 & 3.93810 & 3.48717 & 3.94354 & 3.96366 & & & & & & & 8.4 \\
\hline 0.24 & 3.95317 & 3.93030 & 3.89215 & 3.92882 & & & & & & & & 8.1 \\
\hline 0.25 & 3.92794 & 3.86050 & 3.86050 & 3.89121 & 3.9135 & & & & & & & 7.7 \\
\hline 0.26 & 3.87976 & 3.85599 & 3.81112 & 3.8385 & & & & & & & & 7.4 \\
\hline 0.3 & 3.57455 & 3.54515 & 3.48717 & 3.49684 & 3.52318 & 3.5241 & 3.538 & & 3.548 & & 3.5332 & 6.3 \\
\hline 0.4 & 2.51533 & 2.48139 & 2.39851 & 2.42537 & 2.44774 & 2.4495 & 2.443 & & 2.45 & 2.41 & 2.4631 & 4.2 \\
\hline 0.5 & 1.63988 & 1.59937 & 1.50905 & 1.58692 & 1.60858 & & & 2.01 & 2 & 1.983 & & 3.0 \\
\hline 0.6 & 1.06015 & 1.00273 & 0.92374 & 1.04924 & 1.0741 & 1.6104 & 1.599 & & 1.595 & 1.591 & 1.0941 & 2.2 \\
\hline 0.7 & 0.69344 & 0.56006 & 0.56006 & 0.71258 & 0.74862 & & & & & & 0.7470 & 1.7 \\
\hline 0.74 & 0.5872 & 0.49476 & 0.45770 & 0.6153 & 0.66072 & & & & & & & 1.5 \\
\hline 0.8 & 0.46293 & 0.35111 & 0.33748 & 0.49768 & 0.56512 & 0.5653 & 0.553 & & 0.547 & 0.56 & 0.5544 & 1.3 \\
\hline 0.8544 & 0.37483 & 0.24747 & 0.25537 & 0.41421 & 0.41421 & & & & & & & 1.2 \\
\hline 0.8631 & 0.36099 & 0.23292 & 0.24417 & 0.40224 & 0.68976 & & & & & & & 1.1 \\
\hline 0.8660 & 0.35825 & 0.22819 & 0.24054 & 0.39846 & 0.76223 & & & & & & & 1.1 \\
\hline
\end{tabular}

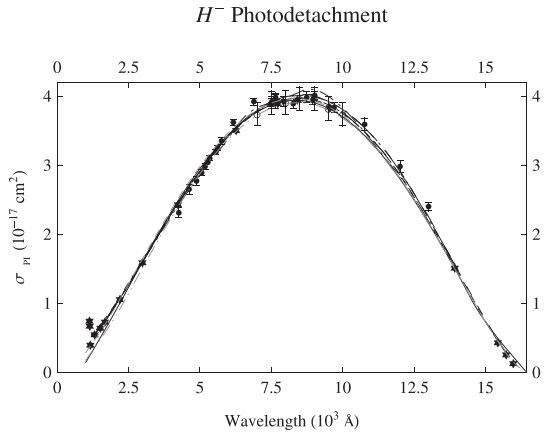

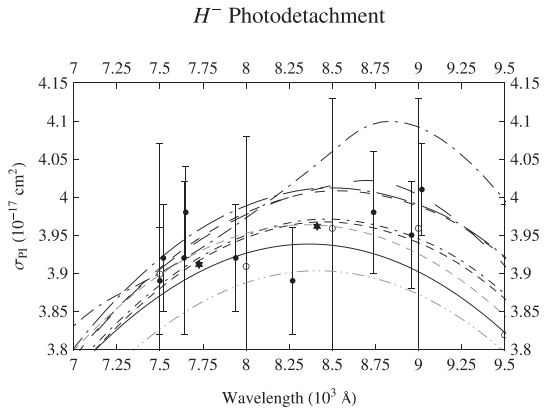

$\mathrm{b}$

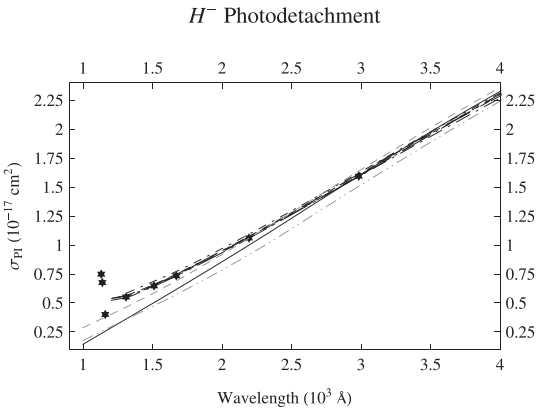

C

Fig. 1. The cross section for photoionizing an electron from the $\left(1 s^{2}{ }^{1} S^{e}\right)$ state of the negative hydrogen ion $\mathrm{H}^{-}(\mathrm{a})$ with the region near the maximum highlighted in (b) and at small wavelengths (high energies) (c). The solid dots with error bars are the experimental results of Smith and Burch [61] normalized at $5280 \AA$ to the average of the values for the 11 theories given in Saha [64], $3.04 \times 10^{-17} \mathrm{~cm}^{2}$. The open circles with error bars are the experimental results of Génévriez and Urbain [62]. The solid line gives the present polarized theoretical result of our three-term polynomial approximation to Bhatia's [20] smooth cutoff function parameter $\mu(k)=0.54-1.46625 k+2.021875 k^{2}$. This was calculated using a 200-term wave function [18] composed of explicitly correlated exponentials. With the gray dot-dot-dashed curve we instead use the cutoff function of Shertzer and Temkin [35]. The gray dashed line is our previous calculation [16] where polarization was not taken into account. The stars are Bhatia's results including polarization and short-range correlations using a 364-term Hylleraas wave function and his cutoff function with the parameter $\mu$ varied for each $k$ to maximize the phase shift. Also shown are a representative sample of other length gauge theories: Venuti and Decleva [63] (small dashed); Saha [64] (medium dashed); Wishart [65] (large dashed); Stewart [66] (large dot dashed); Broad and Reinhardt [67] (medium dot dashed); and Ajmera and Chung [68] (small dot dashed). 


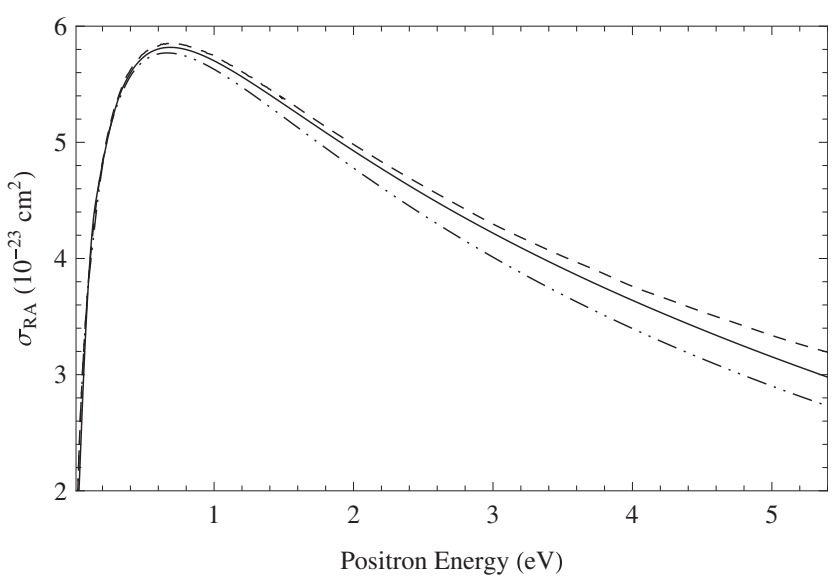

Fig. 2. The cross section for radiatively attaching a second positron to $\overline{\mathrm{H}}(1 s)$ to create the $\left(1 s^{2}{ }^{1} S^{e}\right)$ state of $\overline{\mathrm{H}}^{+}$as a function of positron energy for the present theory (solid line), using a 200-term wave function [18] composed of explicitly correlated exponentials for the now-final state and a polarized now-initial state using our three-term polynomial approximation to Bhatia's smooth cutoff function parameter $\mu(k)=$ $0.54-1.46625 k+2.021875 k^{2}$. The dot-dot-dashed curve is our use of the cutoff function of Shertzer and Temkin [35], and the dashed curve is our previous calculation [16] where polarization was not taken into account.

(iii) Wishart [65] (large dashed) uses a close-coupling pseudostate expansion with the addition of Hylleraastype correlation terms for both the final state and the initial state.

(iv) Stewart [66] (large dot dashed) uses a perturbation method involving Feshbach operators for both the final and the initial states.

(v) Broad and Reinhardt [67] (medium dot dashed) use a CI expansion including pseudostates in the final state and a CI expansion in the initial state.

(vi) Ajmera and Chung [68] (small dot dashed) use a simplified Kohn-Feshbach variational method to find the final state and a Hylleraas correlated wave function with a "tail function" added to it for the initial state.

While our two-electron bound initial state is of the same caliber as the best of these theories, our final state is less so. It is a bound-state orbital that has been polarized by interactions with the a plane wave that multiplies it (5), with the polarization effect spanning all inter-electron distances (13). One might wonder how this first-order perturbation compares with the fourth-order perturbation methods of Stewart [66] or, for that matter, to the nonperturbative methods in this set. If one were to examine an unlabelled version of Figures $1 \mathrm{a}$ and $1 \mathrm{~b}$, there is no indication of anything amiss or anything outstanding in any of the theories, given the size of the error bars in the experimental results of Smith and Burch [61] and of Génévriez and Urbain [62]. One would be pressed to guess which theory was which except at small wavelengths, Figure 1c. Likewise, in an unlabelled version of Table 2 all of the theories differ from each other in the third decimal place, except at a wave vector of 0.5 where three theories are $33 \%$ higher than the other five, and for $k=0.8$ and above, where the first four theories fall progressively farther below the fifth theory.

As we look at the labels of the theories, we see that it is Wishart [65], Stewart [66], and Broad and Reinhardt [67] that are the outliers at $k=0.5$. But it is indeed ours that have only a modest inclusion of short range correlations that are the outliers at $k=0.8$. This confirms the judgment of Burke and Schey [32] and Matese and Oberoi [33] given in Section 3, that it is at higher energies (where $k$ is larger) that short-range effects are expected to dominate. The present work was motivated by the fact that our unpolarized rate coefficient [16] differed significantly from Bhatia's [20] at high temperatures, and one would associate those temperatures with positrons having a larger average momentum and, hence, energy. But one cannot guess how the rate coefficient will vary with temperature from this rough association: one must actually calculate it. Furthermore, our ultimate goal was to give a reliable rate coefficient at low temperatures, but one cannot simply dismiss either long-range or short-range perturbations of the antihydrogen atom without actually doing the calculation to see where the cross-over temperature region, if any, actually lies. Therefore, we do so.

\section{The radiative attachment cross section}

The cross section for radiatively attaching a second positron to $\overline{\mathrm{H}}(1 s)$ to create the $\left(1 s^{2}{ }^{1} S^{e}\right)$ state of the $\overline{\mathrm{H}}^{+}$ ion, via reaction (1), can be obtained from the photoionization cross section via the principle of detailed balance. For this particular case we have [16],

$$
\sigma_{\mathrm{RA}}(k)=\frac{g_{1} p_{\omega}^{2}}{g_{2} p_{e}^{2}} \sigma_{\mathrm{PI}}=\frac{6 \alpha^{2}\left(\hbar^{2} k^{2}+\gamma_{0}^{2}\right)^{2}}{12 \cdot 2^{2} \hbar^{2} k^{2}} \sigma_{\mathrm{PI}},
$$

where $g_{1} / g_{2}=6 / 12$ is the statistical weight ratio. Here the photon momentum relative to the ion is given by $p_{\omega}=$ $\hbar \omega / c=\left(\hbar^{2} k^{2}+\gamma_{0}^{2}\right) / 2 c$, and $p_{e}$ is the positron momentum $\hbar k$. Note that $c$ in atomic units is the inverse of the fine structure constant $\alpha$, and $\hbar=m_{e}=1$.

The radiative attachment cross section, plotted in terms of incoming positron energy $E_{e}=\hbar^{2} k^{2} / 2$ in Figure 2, again shows a moderate downward shift at high energies from our previous results (dashed) when polarization is included (solid) using a three-term polynomial approximation to Bhatia's smooth cutoff function parameter $\mu$. Use of the cutoff function of Shertzer and Temkin [35] (the dot-dot-dashed curve) doubles this downward shift at high energies. Bhatia [20] does not include a table or figures to compare with our radiative attachment cross sections in Figure 2, only for the rate coefficient discussed next, of which it is a part.

In order to estimate formation rates of $\overline{\mathrm{H}}^{+}$it is helpful to calculate positron attachment to $\overline{\mathrm{H}}$ as a function of temperature rather than energy, as is common in astrophysical applications. This rate coefficient $\alpha_{\mathrm{RA}}$ is formed as the expectation value of $v \sigma_{\mathrm{RA}}$ with the normalized 
Table 3. The rate coefficient $\alpha_{\mathrm{RA}}$ for radiatively attaching a second positron to $\overline{\mathrm{H}}(1 s)$ to create the $\left(1 s^{2}{ }^{1} S^{e}\right)$ state of $\overline{\mathrm{H}}^{+}$as a function of temperature in units of $10^{-15} \mathrm{~cm}^{3} / \mathrm{s}$. Column 2 contains our prior unpolarized results [16]. Column 3 contains our current results using three-term polynomial approximation $\mu(k)=0.54-1.46625 k+2.021875 k^{2}$. Column 4 sets $\mu(k)$ to be a constant, 0.5. In column 5 , we have set $\mu(k)$ to vary with $k$ as the exponent: $\mu(k)=0.54 \times 0.18845819320043986^{k}$. Column 6 contains results using the cutoff function of Shertzer and Temkin [35]. These were all calculated with a fully two-positron 200-term wave function [18] composed of explicitly correlated exponentials. The final column contains Bhatia's [20] results including polarization (in which the parameter $\mu$ is varied for each $k$ to maximize the phase shift) and short-range correlations arising from an optical potential, using a 364-term Hylleraas wave function.

\begin{tabular}{|c|c|c|c|c|c|c|}
\hline$T$ & $\begin{array}{l}\text { Prior results } \\
\text { Unpolarized }\end{array}$ & $\begin{array}{l}\text { Present results } \\
\text { Poly. approx. to } \mu\end{array}$ & Constant $\mu$ & $\mu$ varies with $k$ as the exponent & $\mathrm{ST}$ & Bhatia 2013 \\
\hline 1000 & 0.83 & 0.83 & 0.825 & 0.83 & 0.82 & 0.99 \\
\hline 2000 & 1.39 & 1.38 & 1.38 & 1.38 & 1.38 & 1.28 \\
\hline 5000 & 2.39 & 2.37 & 2.36 & 2.37 & 2.34 & 2.40 \\
\hline 7000 & 2.78 & 2.76 & 2.73 & 2.75 & 2.71 & 2.82 \\
\hline 10000 & 3.17 & 3.14 & 3.10 & 3.14 & 3.06 & 3.20 \\
\hline 12000 & 3.36 & 3.31 & 3.27 & 3.32 & 3.22 & 3.37 \\
\hline 15000 & 3.56 & 3.48 & 3.44 & 3.52 & 3.37 & 3.56 \\
\hline 17000 & 3.66 & 3.56 & 3.52 & 3.61 & 3.44 & 3.65 \\
\hline 20000 & 3.77 & 3.64 & 3.60 & 3.72 & 3.50 & 3.75 \\
\hline 22000 & 3.83 & 3.67 & 3.63 & 3.78 & 3.52 & 3.79 \\
\hline 25000 & 3.89 & 3.68 & 3.67 & 3.84 & 3.54 & 3.83 \\
\hline 30000 & 3.96 & 3.66 & 3.68 & 3.90 & 3.52 & 3.83 \\
\hline 35000 & 3.98 & 3.60 & 3.67 & 3.93 & 3.47 & 3.77 \\
\hline 40000 & 3.99 & 3.52 & 3.63 & 3.94 & 3.40 & 3.67 \\
\hline
\end{tabular}

Maxwell-Boltzmann distribution $f(v)$ as,

$$
\begin{aligned}
\alpha_{\mathrm{RA}}(T)= & \left\langle v \sigma_{\mathrm{RA}}\right\rangle=4 \pi \int_{0}^{\infty} d v v \sigma_{\mathrm{RA}} \\
& \times(k(v))\left(\frac{m}{2 \pi k_{\mathrm{B}} T_{e}}\right)^{3 / 2} v^{2} \exp \left[-m v^{2} /\left(2 k_{\mathrm{B}} T_{e}\right)\right] \\
= & \sqrt{\frac{8 k_{\mathrm{B}} T}{m_{e}}} \frac{1}{\sqrt{\pi}} \int_{0}^{\infty} d x x \frac{g_{\mathrm{PI}}}{g_{\mathrm{RA}}} \frac{P_{\omega}^{2}}{p_{e}^{2}} \\
& \times \sigma_{\mathrm{PI}}\left(\sqrt{2 k_{B} T x}\right) \exp [-x] .
\end{aligned}
$$

For temperatures in the range from 5000 to $25000 \mathrm{~K}$, the unpolarized rate coefficient results of our prior work [16] pretty well track Bhatia's [20] results that include both the long-range effects of polarization and short-range correlations. But our unpolarized results continue to climb above his results for temperatures above $25000 \mathrm{~K}$, which may be seen in Table 3 and Figure 3a (dashed curve). Those who may wish to include polarization but have no means to calculate, and maximize, the phase shift have the option of using the cutoff function of Shertzer and Temkin [35], equation (12). One sees in Table 3 and Figure 3a that this (dot-dot-dashed) curve falls well below Bhatia's results at the highest temperatures. One should note that this result includes polarization at all positive distances but not Bhatia's short-range correlations arising from an optical potential.

This significant deviation motivated us to try various functional forms for a cutoff function that might better track Bhatia's by using his general form but with his exponential parameter $\mu$ either a constant, 0.5 , or $\mu(k)$ varying with $k$ as the exponent of a number chosen to correctly give his values at the two published points $k=0$ and $k=0.4$, both with with $h=3$. Bhatia very kindly offered us [38] a third data point to augment his published results for $\mu$ : for $k=0.8$ with $h=3$, he finds $\mu=0.661$. A fit to these points gave us a three-term polynomial approximation $\mu(k)=0.54-1.46625 k+2.021875 k^{2}$. All were calculated with a fully two-positron 200-term wave function [18] composed of explicitly correlated exponentials.

As one might have guessed, the three-term polynomial gave the best results of these approximations: a fairly faithful shape (solid curve) that more closely tracks Bhatia's results than does the curve using Shertzer and Temkin's cutoff function, at a calculational time savings of about a factor of 10. Even the constant- $\mu$ version (dashdash-dotted curve) does pretty well in terms of values near $40000 \mathrm{~K}$, but has a shape more like the unpolarized results.

Setting $\mu(k)$ to vary with $k$ as the exponent, $\mu(k)=$ $0.54 \times 0.18845819320043986^{k}$, gives a result not much different from the unpolarized results, the (dot-dashed) curve. Figure $4 \mathrm{~b}$ provides an explanation of this small polarization effect. At high temperatures, the positron momentum $\hbar k$ will, on average, also be high and the polarization cutoff function for $\mu$ that varies with $k$ as the exponent of a number of order $20 \%$ should still be small for high values near $k=0.8$ (light dot-dashed). Indeed this curve lies far below the other cutoff functions at all values of the positron-atom distance $r_{1}$. As far out at 100 atomic units it is still only allowing a polarization of $40 \%$ of maximum.

On the other hand, the cutoff function of Shertzer and Temkin is the same for all $k$, and furthermore reaches its maximal value at $r_{1}=6$ a.u. It, thus, provides a much more substantial polarization effect at all 


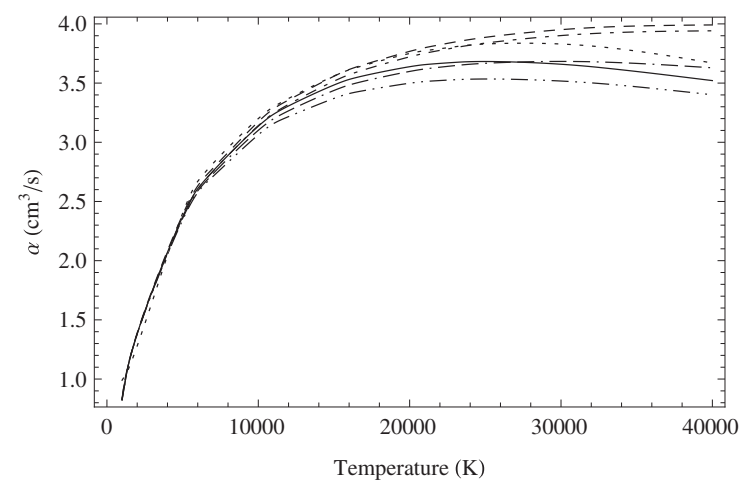

a

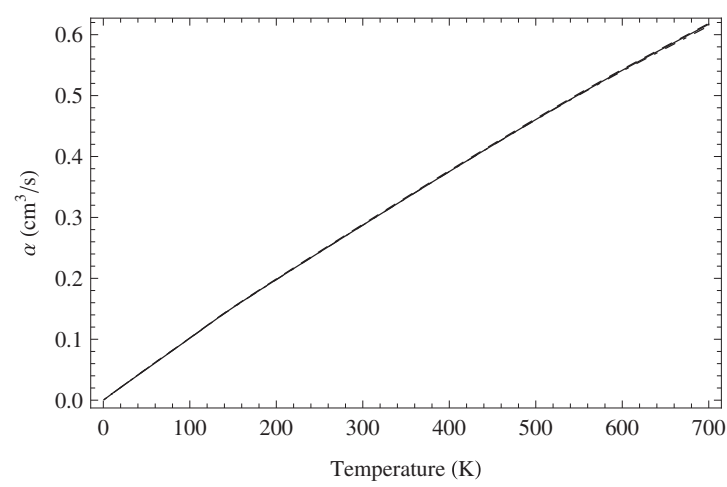

b

Fig. 3. The rate coefficient $\alpha_{\mathrm{RA}}$ for radiatively attaching a second positron to $\overline{\mathrm{H}}(1 s)$ to create the $\left(1 s^{2}{ }^{1} S^{e}\right)$ state of $\overline{\mathrm{H}}^{+}$as a function of temperature in units of $10^{-15} \mathrm{~cm}^{3} / \mathrm{s}$. (a) The solid curve gives the present theory (solid line) using a three-term polynomial approximation for $\mu$ fit to Bhatia's data points $\mu=0.54$ at $k=0$ [36], $\mu=0.277$ at $k=0.4$ [37], and $\mu=0.661$ for $k=0.8$ [38], all with $h=3: \mu(k)=0.54-1.46625 k+2.021875 k^{2}$. The dashed curve gives our prior unpolarized results [16] and the similarly shaped dot-dashed curve results from setting $\mu(k)$ to vary with $k$ as the exponent: $\mu(k)=0.54 \times 0.18845819320043986^{k}$. The dash-dash-dotted curve has $\mu(k)$ set to a constant, 0.5 (with $h=3$ ). Finally, the dot-dot-dashed curve shows results using the cutoff function of Shertzer and Temkin [35]. The small-dotted curve is interpolated from Bhatia's Table IX [20] including polarization and short-range correlations, in which he uses a 364-term Hylleraas wave function and his cutoff function with the parameter $\mu$ varied for each $k$ to maximize the phase shift. For low temperatures (b), the rate coefficient increases nearly linearly with $T$ and the polarized results (solid line) using the three-term polynomial approximation are little distinguishable from its competitors or even the unpolarized results (dashed line).

temperatures than our three model cutoff functions. We see in Table 3 however, that at low temperatures the polarization effect is damped for all models. Thus, it is only at higher temperatures that this enhanced polarization effect for the Shertzer and Temkin cutoff function becomes obvious.

\section{Low-temperature results}

At the lowest temperature Bhatia calculated, $1000 \mathrm{~K}$, our results are consistently $16 \%$ lower than his and agree with each other to three decimal places. Even the polarized results using Shertzer and Temkin smoothly join our results, showing that polarization has lost its power as the temperature drops. One would not expect that the 200-term wave function [18] we used, composed of explicitly correlated exponentials of the kind introduced by Thakkar and Smith [19], would be significantly inferior to Bhatia's [20] 364-term Hylleraas wave function. It is possible that this discrepancy is due to our only modest inclusion of short-range correlations versus Bhatia's robust inclusion. A more prosaic possibility is that there is a nonconvergence in the numerical integration of our results or his. Indeed we found that we had to use Adaptive Monte Carlo numerical integration to get consistent results for temperatures below $700 \mathrm{~K}$, but at $1000 \mathrm{~K}$ all of our numerically approaches were consistent with each other.

The integrand is akin to a slightly-rounded Heaviside step function and is difficult to handle numerically, so we wished to have an analytic check on our results. In an earlier paper we gave a result [15] using Ohmura and Ohmura's [17] effective range theory, where the final state was reduced to a one-electron wave function, giving a much less complicated matrix element than that resulting from equation (14):

$$
\mu_{\mathrm{ER}}=8 \pi \frac{C k}{\left(k^{2}+\gamma_{0}^{2}\right)^{2}},
$$

where the constant $C=0.315878$ [69] has its origin in the $H^{-}$wave function and $\gamma_{0}=0.2355883$ a.u. [27] is related to the electron affinity, the energy difference between the initial and final bound states, by $I=\gamma_{0}^{2} / 2$. We were able to evaluate this integral analytically for this simplified matrix element giving

$$
\begin{aligned}
\alpha_{\mathrm{ER}}= & \left\langle v \sigma_{\mathrm{RAER}}\right\rangle \\
= & 1.213 \times 10^{-22} \mathrm{~cm}^{2}\left(\frac{m}{2 \pi}\right)^{3 / 2} \frac{8 \pi}{m^{2}} \sqrt{E_{\mathrm{B}}} \\
& \times \int_{0}^{\infty} d x \frac{x^{3 / 2}}{x+\frac{E_{\mathrm{B}}}{k_{\mathrm{B}} T}} e^{-x} \\
= & 9.378 \times 10^{-15} \frac{\mathrm{cm}^{3}}{\mathrm{~s}}\left(\frac{I}{k_{\mathrm{B}} T}\right)^{3 / 2} e^{\frac{I}{k_{\mathrm{B}} T}} \Gamma\left(-\frac{3}{2}, \frac{I}{k_{\mathrm{B}} T}\right) .
\end{aligned}
$$

For $T=1000 \mathrm{~K}$, this gives $0.85 \times 10^{-15} \mathrm{~cm}^{3} / \mathrm{s}$, matching the numerical integral to five decimal places. Comparison in our later paper [16] using a fully two-electron wave function (composed of explicitly correlated exponentials) found that the effective range theory gave results about $4 \%$ too high. This accounts for the modestly higher $0.85 \times 10^{-15} \mathrm{~cm}^{3} / \mathrm{s}$ when compared to the $0.83 \times 10^{-15} \mathrm{~cm}^{3} / \mathrm{s}$ results of the present theories in the first line of Table 3. We take this as confirmation of the convergence of our numerical integrals. 

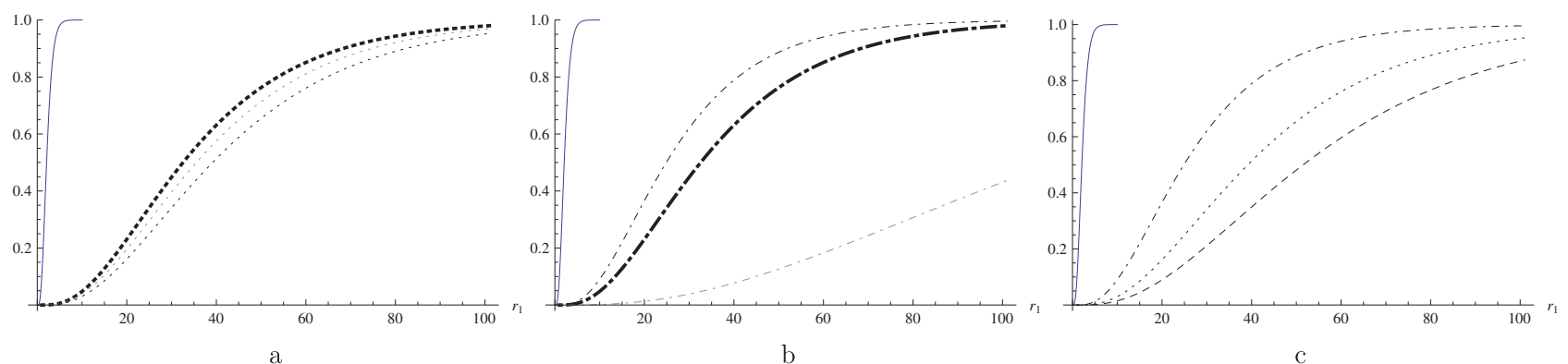

Fig. 4. The four model cutoff functions at (a) $k=0.1$ as a function of the positron-atom distance $r_{1}$. The solid line is the cutoff function of Shertzer and Temkin [35] (12), which is the same for all $k$, as is $\mu$ set to the constant, 0.5 (dark dotted). Using $\mu(k)$ set to vary with $k$ as the exponent, $\mu(k)=0.54 \times 0.18845819320043986^{k}$, gives the light dotted curve, and the three-term polynomial approximation $\mu(k)=0.54-1.46625 k+2.021875 k^{2}$ is the medium dotted curve. The four cutoff functions at $k=0.8$ (b): Shertzer and Temkin (solid line), constant $\mu$ (dark dot-dashed), $\mu$ with $k$ as the exponent (light dot-dashed), and the three-term polynomial approximation (medium dot-dashed). The three-term polynomial approximation for the three momenta $k=0.1$ (dotted), $k=0.5$ (dashed), and $k=0.8$ (dot-dashed) are shown together in (c), along with the cutoff function of Shertzer and Temkin.

Although the exposition of the technique would be a full paper in itself, we have likewise been able to analytically integrate the non-polarized results for our full two-electron wave function composed of explicitly correlated exponentials and they likewise agree with the corresponding numerical integrals. One would thus entertain the possibility that either Bhatia's results are not fully converged at these lowest of his energies, that there is something like a remaining sensitivity of his results on a finite substitute for the infinite upper limit on his numerical integral, or that the effects of Bhatia's short-range more robust short-range correlations arising from an optical potential have not died out with low temperature as rapidly as the polarization contributions. Even if the latter is the case at $1000 \mathrm{~K}$, one would expect the trend of all of the curves merging to continue as the temperatures drop further, so we believe our very-low temperature results should not change much were Bhatia's short-range correlations included.

Figure 3b shows our results for temperatures below Bhatia's lowest tabled temperature, $1000 \mathrm{~K}$. It also shows the near linear $T$-dependence of the rate coefficients in all approximations. For $T \lesssim 6 \mathrm{~K}$ the rate coefficient for the three-term polynomial approximation for $\mu$ may be fit by $\alpha_{\mathrm{RA}}=0.00104 \times 10^{-15} \mathrm{~cm}^{3} \mathrm{~s}^{-1} T \mathrm{~K}^{-1}$.

\section{Conclusions}

We have reduced to analytic form the six-dimensional, three body integrals containing the transition amplitude for radiatively attaching a second positron to a polarized $\overline{\mathrm{H}}(1 s)$ atom to create the $\left(1 s^{2}{ }^{1} S^{e}\right)$ state of $\overline{\mathrm{H}}^{+}$. Whereas conventional means to integrate the polarization cross-term has every appearance of being singular at the origin, but non-singular when integrated numerically, one may show that this final integral has two singular terms that may be made to cancel in lowest order. However, such approaches leave one without an analytical result for the remaining nonsingular part of the integral. We instead used an integro-differential transform based on Gaussian transforms to bypass this blockage to yield a fully analytic result. Our results apply to twopositron/electron wave functions composed of explicitly correlated exponentials.

This approach will be key to any extension to Hylleraas wave functions, since the above explicitly correlated exponentials comprise the term with no powers within Hylleraas coordinates. That is the one Hylleraas term that contains singularities in conventional approaches, and is circumvented herein. The integrals for the remaining, arbitrary-powered Hylleraas terms may be found in a straightforward way by extending Keating's method $[16,39]$.

This analytic result means that the subsequent numerical integral (48) to find the rate coefficient $\alpha_{\mathrm{RA}}$ may be performed on a laptop or desktop computer rather than requiring massive computing power. Most results required 20 min per data point on a 2013-era computer using Mathematica, and even the results using the cutoff function of Shertzer and Temkin [35] required just an overnight run per data point.

While Bhatia's [20] polarization cutoff function (13) may be considered the gold standard since it has has a parameter $\mu$ that varies for each $k$ to maximize the phase shift, those with no means to calculate these phase shifts need an alternative. We crafted a three-term polynomial approximation for $\mu$ fit to his data points at $k=0[36]$, $k=0.4$ [37], and $k=0.8$ [38], all with $h=3(\mu(k)=$ $\left.0.54-1.46625 k+2.021875 k^{2}\right)$ that gave results reasonably close results to Bhatia's rate coefficient $\alpha_{\mathrm{RA}}$ up through $40000 \mathrm{~K}$. In contrast, the cutoff function of Shertzer and Temkin [35] gave results that were farther from Bhatia's throughout the upper temperature range. One should note that neither result includes Bhatia's short-range correlations arising from an optical potential.

Finally, we extend the polarization results below Bhatia's lowest value of $1000 \mathrm{~K}$ (for the equivalent matter problem) to the low temperatures required for the GBAR collaboration's studies of the gravitational interaction of the anti-atom. We find that the effects of polarization are considerably muted at these temperatures 
and would expect the same of Bhatia's short-range correlations.

Given our assumption that Bhatia's short-range correlations will be suppressed at very low temperatures, just as we have shown the polarization contributions to have been, we estimate that reaction rates will be below $5 \times 10^{-7} \mathrm{~s}^{-1}$ per antihydrogen atom [15]. That means that if all ALPHA's antiprotons could be converted into trapped $\overline{\mathrm{H}} \mathrm{s}$, while still allowing the anti-atoms to interact with warm positron clouds, the $\overline{\mathrm{H}}^{+}$rates would nevertheless be lower than $5 \times 10^{-3} \mathrm{~s}^{-1}$, which might just be observable, given the long antihydrogen storage times achieved by ALPHA [8].

We are deeply grateful to Dr. Anand Bhatia for providing us with his calculated results for the cutoff function parameter at $k=0.8$.

\section{Author contribution statement}

The first author contributed the analytical result for the most difficult of the two polarization terms and, in particular, the key insight of performing the $s$ integral last, as in equation (42), to avoid the persistently singular terms. The second author contributed the analytical result for the other polarization term and performed the numerical calculations.

Publisher's Note The EPJ Publishers remain neutral with regard to jurisdictional claims in published maps and institutional affiliations.

\section{References}

1. S. Maury, Hyperfine Interact. 109, 43 (1997)

2. M. Amoretti et al. (ATHENA Collaboration), Nature 419, 456 (2002)

3. G. Gabrielse et al. (ATRAP Collaboration), Phys. Rev. Lett. 89, 213401 (2002)

4. Y. Enomoto et al., Phys. Rev. Lett. 105, 243401 (2010)

5. G.B. Andresen et al. (ALPHA Collaboration), Nature 468, 456 (2010)

6. G.B. Andresen et al. (ALPHA Collaboration), Phys. Lett. B 695, 95 (2011)

7. G. Gabrielse et al. (ATRAP Collaboration), Phys. Rev. Lett. 108, 113002 (2012)

8. G.B. Andresen et al. (ALPHA Collaboration), Nat. Phys. 7, $558(2011)$

9. C. Amole et al. (ALPHA Collaboration), Nature 483, 439 (2012)

10. C. Amole et al. (ALPHA Collaboration), Nat. Commun. 5, 3955 (2014)

11. The ALPHA Collaboration, A.E. Charman, Nat. Commun. 4, 1875 (2013)

12. J. Walz, T.W. Hänsch, Gen. Relativ. Gravitation 36, 561 (2004)

13. P. Pérez, Y. Sacquin, Classical Quantum Gravity 29, 184008 (2012)

14. D.P. van der Werf, Int. J. Mod. Phys. Conf. Ser. 30, 1460263 (2014)
15. C.M. Keating, M. Charlton, J.C. Straton, J. Phys. B: At. Mol. Opt. Phys. 47, 225202 (2014)

16. C.M. Keating, K.Y. Pak, J.C. Straton, J. Phys. B: At. Mol. Opt. Phys. 49, 074002 (2016)

17. T. Ohmura, H. Ohmura, Phys. Rev. 118, 154 (1960)

18. N.M. Cann, Ph.D. thesis, Dalhousie University, Halifax, Canada, 1993, p. 28, Table 2.3.4; dalspace.library.dal. ca/bitstream/handle/10222/55378/NN93638.PDF

19. A.J. Thakkar, V.H. Smith, Phys. Rev. A 15, 1 (1977)

20. A.K. Bhatia, Phys. Rev. A 87, 042705 (2013)

21. L.D. Landau, E.M. Lifshitz, in Quantum Mechanics (Non-relativistic Theory), Course of Theoretical Physics (Pergamon Press, Oxford, 1977), Vol. 3, p. 606

22. G.W.F. Drake, Astrophys. J. 189, 161 (1974)

23. V.L. Jacobs, A.K. Bhatia, A. Temkin, Astrophys. J. 242, 1278 (1980)

24. S. Chandrasekhar, Astrophys. J. 102, 223 (1945)

25. S. Chandrasekhar, D.D. Elbert, Astrophys. J. 128, 633 (1958)

26. A. Ghoshal, Y.K. Ho, Phys. Rev. E 81, 016403 (2010)

27. C.L. Pekeris, Phys. Rev. 112, 1649 (1958)

28. C.L. Pekeris, Phys. Rev. 126, 1470 (1962)

29. A. Temkin, Phys. Rev. 107, 1004 (1957)

30. S.J. Ward, M.R.C. McDowell, J.W. Humberston, Europhys. Lett. 1, 167 (1986)

31. A. Temkin, Phys. Rev. 116, 358 (1959)

32. P.G. Burke, H.M. Schey, Phys. Rev. 126, 147 (1962)

33. J.J. Matese, R.S. Oberoi, Phys. Rev. A 4, 569 (1971)

34. P.G. Burke, D.F. Gallaher, S. Geltman, J. Phys. B: At. Mol. Opt. Phys. 2, 1142 (1969)

35. J. Shertzer, A. Temkin, Phys. Rev. A 74, 052701 (2006)

36. A.K. Bhatia, Phys. Rev. A 75, 032713 (2007)

37. A.K. Bhatia, Atoms 7, 17 (2017)

38. A.K. Bhatia, personal communication (2019)

39. C.M. Keating, A method for achieving analytic formulas for three body integrals consisting of powers and exponentials in all three interparticle hyllerass coordinates, M.Sc. thesis, Portland State University, 2015. http://archives . $\mathrm{pdx} . \mathrm{edu} / \mathrm{ds} / \mathrm{psu} / 16438$

40. E.A. Hyllerass, Z. Phys. 54, 347 (1929)

41. E. Ley-Koo, C.F. Bunge, Phys. Rev. A 40, 1215 (1989)

42. C.J. Joachain, in Quantum Collision Theory (NorthHolland, NY, 1983), p. 671, Eq. (B.44)

43. W. Magnus, F. Oberhettinger, R.P. Soni, in Formulas and Theorems for the Special Functions of Mathematical Physics, 3rd edition (Springer-Verlag, Heidelberg, New York, 1966), p. 107

44. M. Abramowitz, I.A. Stegun (Eds.), in Handbook of Mathematical Functions with Formulas, Graphs, and Mathematical Tables (National Bureau of Standards, Applied Mathematics Series, 55, Washington, 1970), p. 798 Table 22.9

45. E.W. Weisstein, Legendre polynomial. From MathWorld http://mathworld. wolfram. com/LegendrePolynomial. html

46. J.C. Straton, Phys. Rev. A 41, 71 (1990)

47. I.S. Gradshteyn, I.M. Ryzhik, in Table of Integrals, Series, and Products, 5th edition (Academic, New York, 1994), p. 382 , No. 3.461 .2

48. I.S. Gradshteyn, I.M. Ryzhik, in Table of Integrals, Series, and Products, 5th edition (Academic, New York, 1994), p. 384 , No. 3.471 .9

49. I.M. Cheshire, Proc. Phys. Soc. 83, 227 (1964) 
50. C.J. Joachain, in Quantum Collision Theory (NorthHolland, NY, 1983), p. 49, Eq. (3.27)

51. M.R.C. McDowell, J.P. Coleman, in Introduction to the Theory of Ion-Atom Collisions (North-Holland, 1970), p. 221, Eq. (5.1.2)

52. N.F. Mott, H.S.U. Massey, in The Theory of Atomic Collisions, 3rd edition (Oxford, 1965), p. 133, Eq. (84)

53. C.J. Joachain, in Quantum Collision Theory (NorthHolland, NY, 1983), p. 69, Eq. (4.44)

54. M.R.C. McDowell, J.P. Coleman, in Introduction to the Theory of Ion-Atom Collisions (North-Holland, 1970), p. 76, Eq. (A2.1.2)

55. N.F. Mott, H.S.U. Massey, in The Theory of Atomic Collisions, 3rd edition (Oxford, 1965), p. 23, Eq. (15)

56. C.J. Joachain, in Quantum Collision Theory (NorthHolland, NY, 1983), p. 71, Eq. (4.63)

57. M.R.C. McDowell, J.P. Coleman, in Introduction to the Theory of Ion-Atom Collisions (North-Holland, 1970), p. 74, Eq. (2.8.5)
58. N.F. Mott, H.S.U. Massey, in The Theory of Atomic Collisions, 3rd edition (Oxford, 1965), p. 24, Eq. (17)

59. H. Feshbach, Ann. Phys. 19, 287 (1962)

60. A.K. Bhatia, Atoms 6, 27 (2018)

61. S.J. Smith, D.S. Burch, Phys. Rev. 116, 1125 (1959)

62. M. Génévriez, X. Urbain, Phys. Rev. A 91, 033403 (2015)

63. M. Venuti, P. Decleva, J. Phys. B: At. Mol. Opt. Phys. 30, 4839 (1997)

64. H.P. Saha, Phys. Rev. A 38, 4546 (1988)

65. A.W. Wishart, J. Phys. B: At. Mol. Opt. Phys. 12, 3511 (1979)

66. A.L. Stewart, J. Phys. B: At. Mol. Opt. Phys. 11, 3851 (1978)

67. J.T. Broad, W.P. Reinhardt, Phys. Rev. A 14, 2159 (1976)

68. M.P. Ajmera, K.T. Chung, Phys. Rev. A 12, 475 (1975)

69. A.M. Frolov, J. Phys. B: At. Mol. Opt. Phys. 37, 853 (2004) 